\title{
Objectives and Constraints for Wind Turbine Optimization
}

\author{
Andrew Ning \\ Brigham Young University, aning@byu.edu \\ Rick Damiani \\ National Renewable Energy Laboratory \\ Patrick Moriarty \\ National Renewable Energy Laboratory
}

Follow this and additional works at: https://scholarsarchive.byu.edu/facpub

Part of the Mechanical Engineering Commons

\section{Original Publication Citation}

Ning, A., Damiani, R., and Moriarty, P., "Objectives and Constraints for Wind Turbine Optimization," ASME Wind Energy Symposium, Grapevine, TX, Feb. 2013. doi:10.2514/ 6.2013-201

\section{BYU ScholarsArchive Citation}

Ning, Andrew; Damiani, Rick; and Moriarty, Patrick, "Objectives and Constraints for Wind Turbine Optimization" (2013). Faculty Publications. 1728.

https://scholarsarchive.byu.edu/facpub/1728

This Conference Paper is brought to you for free and open access by BYU ScholarsArchive. It has been accepted for inclusion in Faculty Publications by an authorized administrator of BYU ScholarsArchive. For more information, please contact ellen_amatangelo@byu.edu. 


\title{
Objectives and Constraints for Wind Turbine Optimization
}

\author{
S. Andrew Ning*, Rick Damiani ${ }^{\dagger}$, and Patrick J. Moriarty ${ }^{\dagger}$ \\ National Renewable Energy Laboratory, Golden, Colorado, 80401
}

\begin{abstract}
Efficient extraction of wind energy is a complex multidisciplinary process. This paper examines common objectives used in wind turbine optimization problems. The focus is not on the specific optimized designs, but rather on understanding when certain objectives and constraints are necessary, and what their limitations are. Maximizing annual energy production, or even using sequential aero/structural optimization, is shown to be significantly suboptimal compared to integrated aero/structural metrics. Minimizing the ratio of turbine mass to annual energy production can be effective for fixed rotor diameter designs, as long as the tower mass is estimated carefully. For variable diameter designs, the predicted optimal diameter may be misleading. This is because the mass of the tower dominates the total turbine mass, but the cost of the tower is a much smaller fraction of overall turbine costs. Minimizing cost of energy is a much better metric, though high fidelity in the cost modeling is as important as high fidelity in the physics modeling. Furthermore, deterministic cost of energy minimization can be inadequate, given the stochastic nature of the wind and various uncertainties associated with physical processes and model choices. Optimization in the presence of uncertainty is necessary to create robust turbine designs.
\end{abstract}

\section{Nomenclature}

$\begin{array}{ll}A & =\text { area } \\ \mathrm{AEP} & =\text { annual energy production } \\ \mathrm{BOS} & =\text { balance-of-station } \\ \mathrm{COE} & =\text { cost of energy } \\ C_{T} & =\text { thrust coefficient } \\ D & =\text { modor diameter } \\ E & =\text { fixed charge rate } \\ \mathrm{FCR} & \text { height of tower } \\ H & =\text { area moment of inertia } \\ I & =\text { benjective } \\ J & =\text { moment about axes } i \\ M_{b} & \text { number of cycles } \\ M_{i} & =\text { axial force in direction of axes } i \\ N & =\text { operations and maintenance } \\ N_{i} & \text { power } \\ \mathrm{O \& M} & \text { fatigue stress } \\ P & =\text { planform area } \\ S_{f} & =\text { rotor thrust } \\ S_{p l a n} & \text { turbine capital costs } \\ T & \text { freestream velocity } \\ U_{\infty} & =\end{array}$

*Postdoctoral Researcher, National Wind Technology Center, 15013 Denver West Parkway, MS3811, andrew.ning@nrel.gov, AIAA Member

†Senior Engineer, National Wind Technology Center, 15013 Denver West Parkway, MS3811, AIAA Member 


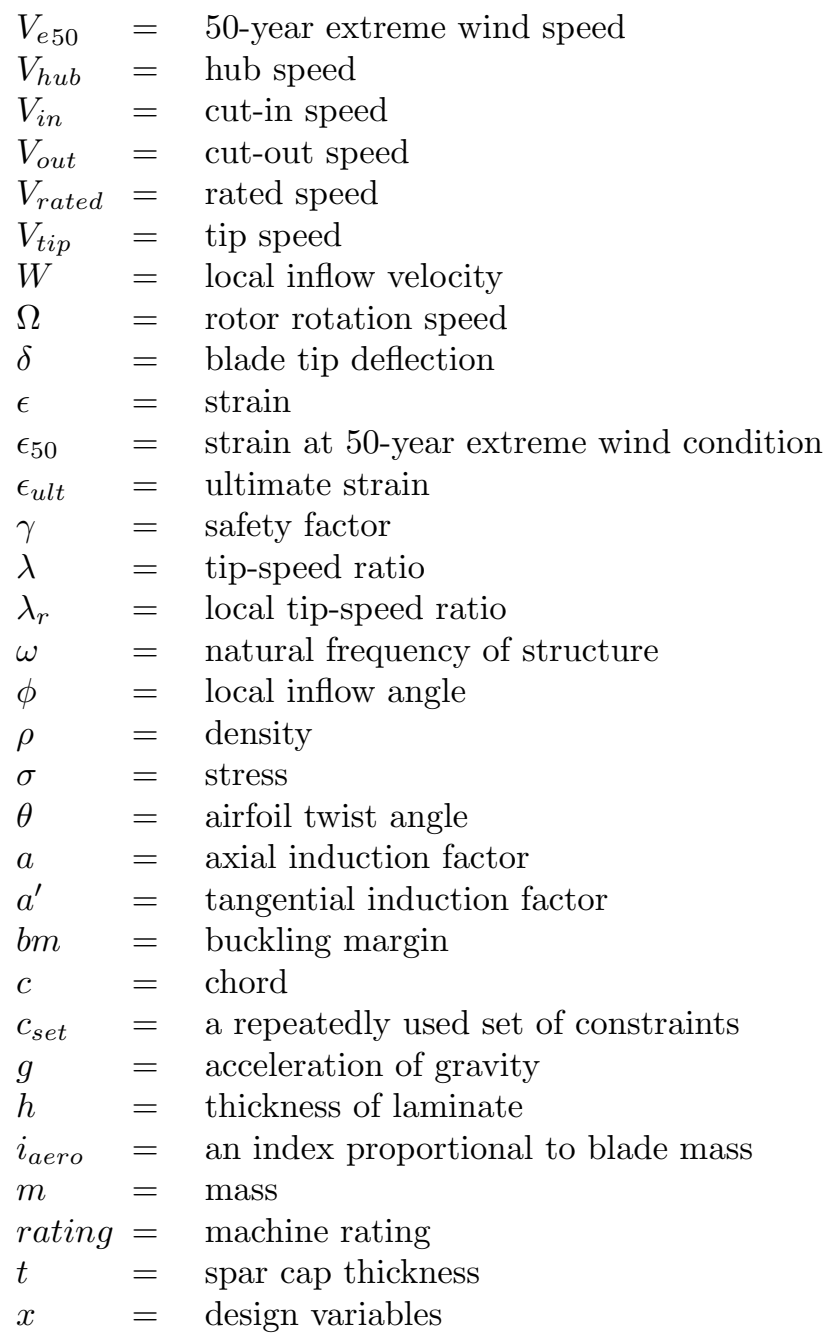

Subscripts

$0=$ quantity of the reference model

cr $\quad=$ critical

mid $=$ evaluated at midpoint of tower

$R N A=$ rotor $/$ nacelle assembly

\section{Superscripts}

$\bar{x}=$ a quantity normalized relative to the reference design (e.g., $\left.\bar{x}=x / x_{0}\right)$

\section{Introduction}

Increasing global energy needs and greater awareness of the benefits of renewable sources has driven renewed interest in wind energy. Harvesting wind energy efficiently is a complex process that requires a multidisciplinary effort in wind turbine design, site selection, and plant layout. Many trade-offs exist in aerodynamic performance, structural efficiency, land-use footprint, operational versus manufacturing and maintenance costs, and so on. Therefore, multidisciplinary optimization and uncertainty analysis are important tools to evaluate design choices and further improve the economics of wind energy. A number of previous studies have examined optimization of wind turbines using a wide variety of approaches. In these studies, design variables ranged from descriptions of only the rotor blade to descriptions of complete turbines. Model fidelity included simple analytic models, time-domain unsteady aeroelastic calculations, and three-dimensional computational fluid dynamics with structural finite element analyses. Objectives included maximum annual energy production (AEP), multiobjective maximum power and minimum blade root bending moment, and minimum cost of energy. Optimization approaches included gradient-based methods, 
direct-search methods, and multilevel methods. A select number of these studies are highlighted below to provide examples of the variety of approaches used.

Fuglsang and Madsen optimized a rotor using time-domain aeroelastic calculations. Their study used cost of energy as the objective and aerodynamic noise as a constraint. Airfoil optimization was treated by modifying the lift and drag curves directly; focusing on the maximum lift coefficient. ${ }^{1}$ Diveux et al. optimized a combined rotor/drivetrain model for minimum annual energy cost using a simplified aerodynamic performance model that combined an analytic estimation of maximum power coefficient with a parameterized power curve. They used a genetic algorithm and their optimization studies focused on the benefits of sitespecific optimization. ${ }^{2}$ Fuglsang et al. examined site-specific optimization across a broader range of conditions using an aeroelastic turbine model and an objective of minimum levelized cost of energy. ${ }^{3,4}$ Kenway and Martins optimized blade and airfoil shape for maximum annual energy production in a coupled aeroelastic analysis using a blade element momentum method and a finite element model. A gradient-based approach with finite differences estimated using the complex-step method was used to ensure robust convergence. ${ }^{5}$ Petrone et al. combined uncertainty analysis with optimization to examine the sensitivity of optimized turbines to uncertainties caused by insect contamination. Their optimization approach employed a genetic algorithm in a framework composed of existing National Renewable Energy Laboratory (NREL) tools. ${ }^{6}$ Maki et al. used a multidisciplinary optimization approach where two discipline-specific objectives were pursued and the system-level optimization minimized the cost of energy. Design variables focused on the rotor, and the aerodynamic and structural disciplines used metamodels generated from analysis tools developed at NREL. ${ }^{7}$ Bottasso et al. maximized AEP per turbine weight in a multifidelity aerodynamic and structural optimization framework. ${ }^{8}$

This paper approaches the turbine optimization problem with a different focus than the previous studies. The purpose of this study is not to demonstrate a specific methodology, or even to present optimized wind turbines per se, but rather to understand how different choices in the optimization problem and model choices impact the quality of the solutions. The primary goal is to better understand how different objectives affect the optimal solution. To facilitate this understanding, the models used should: capture the fundamental trade-offs in the physics, execute rapidly to allow for a wide range of design studies, and converge robustly and with high accuracy to allow for fair comparisons in the designs. To this end, simple physics-based models were developed that produce smooth output ( $C^{1}$ continuous) to allow for reliable gradient estimation. The optimization studies of this work concentrate on the design of the rotor blades, but the impact on resizing the rest of the turbine as well as plant-level costs was included. The work conducted was part of a larger effort at NREL to apply systems engineering techniques to wind energy applications. ${ }^{9}$

The following section describes the methodology, which includes the rotor aerodynamic analysis, rotor structural analysis, cost model, reference model, and optimization strategy used. Next, a number of optimization studies are presented. These studies examined the important considerations in maximizing annual energy production, minimizing the ratio of turbine mass to annual energy production, and minimizing cost of energy.

\section{Methodology}

Although the impact on the entire turbine was considered, the focus of this study was on optimizing the rotor blades. Thus, the main thrust of the methodology was the development of appropriate aerodynamic and structural analysis tools for the blades. The effect of hub, nacelle, and foundation sizing was handled through simpler scaling relations. A modified version of the NREL cost and scaling model ${ }^{10}$ was $_{\text {used to }}$ predict the cost of energy. The physics-based models were implemented in C++ and Fortran, and were linked together in a common framework in Python. This approach retained most of the speed advantage of the compiled languages, but allowed for high flexibility in an object-oriented environment. The computational efficiency and flexibility was important to allow for both a large number and wide variety of studies.

\section{II.A. Rotor Aerodynamics}

\section{II.A.1. Blade Element Momentum Method}

The rotor aerodynamic analysis was based on blade element momentum (BEM) theory. Using BEM theory in a gradient-based rotor optimization problem can be challenging because of occasional convergence difficulties of the BEM equations. The standard approach to solving the BEM equations is to arrange the equations as 
functions of the axial and tangential induction factors and solve the fixed-point problem:

$$
\left(a, a^{\prime}\right)=f_{f p}\left(a, a^{\prime}\right)
$$

using either fixed-point iteration, Newton's method, or a related fixed-point algorithm. An alternative approach is to use nonlinear optimization to minimize the sum of the squares of the residuals of the induction factors (or normal and tangential loads). Although these approaches are generally successful, they do suffer from instabilities and failure to converge in some regions of the design space, ${ }^{11}$ and thus require increased complexity ${ }^{12}$ and/or heuristics (and may still not always converge).

Ning ${ }^{13}$ recently developed a new solution methodology to the BEM equations that offers guaranteed convergence properties. When used with a smooth $\left(C^{1}\right.$ continuous $)$ description of the airfoil force coefficients, this method is particularly advantageous for use in gradient-based optimization applications. Only a summary of the method is presented here; further details are discussed in Ref. 13.

The new BEM methodology transforms the two-variable, fixed-point problem into an equivalent onedimensional root-finding problem. This is enormously beneficial as methods exist for one-dimensional rootfinding problems that are guaranteed to converge as long as an appropriate bracket can be found. The key insight to this reduction is to use the local inflow angle $(\phi)$ and the magnitude of the inflow velocity $(W)$ as the two unknowns in specifying the inflow conditions, rather than the traditionally used axial and tangential induction factors (see Figure 1).

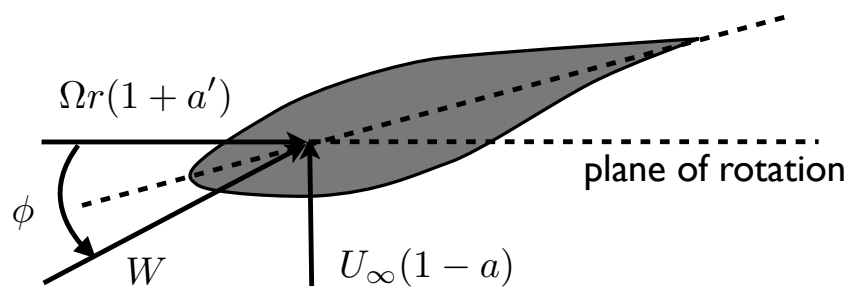

Figure 1. Parameters specifying inflow conditions of a rotating blade section.

This approach allows the BEM equations to be reduced to a one-dimensional residual function as a function of $\phi$ :

$$
f(\phi)=\frac{\sin \phi}{1-a(\phi)}-\frac{\cos \phi}{\lambda_{r}\left(1+a^{\prime}(\phi)\right)}=0
$$

Figure 2 shows the typical behavior of $f(\phi)$ over the range $\phi \in(0, \pi / 2]$. Almost all solutions for wind turbines fall within this range (for the provable convergence properties to be true, solutions outside of this range must also be considered; see Ref. 13 for details). Ref. 13 demonstrates through mathematical proof that the methodology will always find a bracket to a zero of $f(\phi)$, without any singularities in the interior. This proof, along with existing proofs for root-finding methods like Brent's method, ${ }^{14}$ implies that a solution is guaranteed to be found. Furthermore, not only is the solution guaranteed to be found, but it can be found efficiently and in a continuous manner. This behavior allows gradient-based algorithms to be used to solve the rotor optimization problem much more effectively than with traditional BEM solution approaches.

Any corrections to the BEM method can be used with this methodology (e.g., finite number of blades and skewed wake) as long as the axial induction factor can be expressed as a function of $\phi$ (either explicitly or through a numerical solution). This particular implementation chooses to include both hub and tip losses using Prandtl's method, ${ }^{15}$ and a high-induction factor correction by Buhl. ${ }^{16}$ Drag is included in the computation of the induction factors. For a given wind speed, a spline is fit to the normal and tangential forces along the radial discretization of the blade before integrating for thrust and torque. This allows for smoother variation in thrust and torque for improved gradient estimation. For computational efficiency, portions of the methodology that are used heavily are implemented in Fortran and called as a module in Python using f2py. The resultant BEM code is called CCBlade (C - continuity, $\mathrm{C}$ - convergence). 


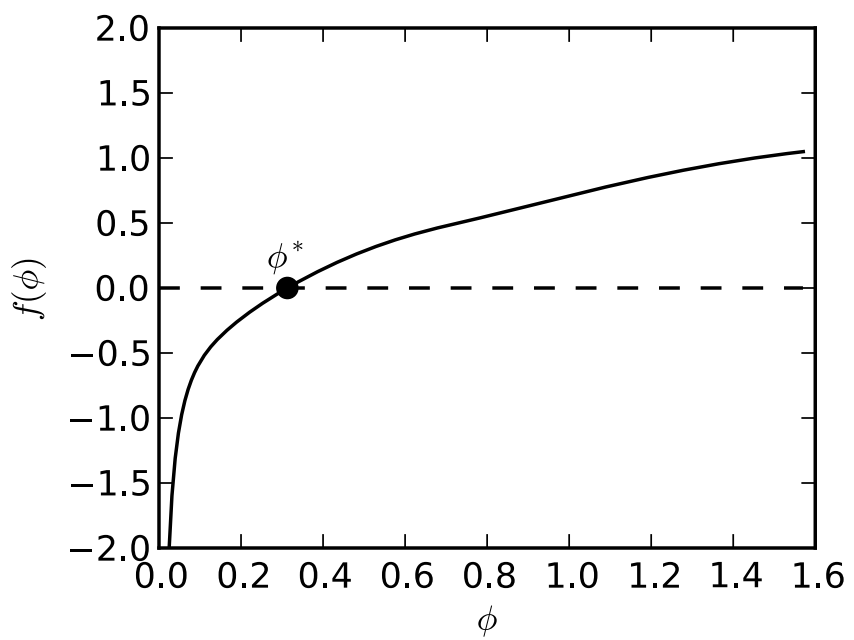

Figure 2. Residual function of BEM equations using Ning's methodology. ${ }^{13}$ Solution point is where $f(\phi)=0$.

\section{II.A.2. Airfoil Section Analysis}

For the airfoil sectional analysis, airfoil force coefficients may be directly supplied covering the full angle of attack range from $-180^{\circ}$ to $180^{\circ}$, or 2 -dimensional airfoil data can be supplied which is subsequently corrected for rotational effects and extrapolated to the full angle of attack range. For the latter case, the corrections were not applied a priori for a given section, but rather re-computed each time the rotor geometry was modified. Three-dimensional rotational corrections were applied using the Du-Selig method ${ }^{17}$ for lift and the Eggers method ${ }^{18}$ for drag. Next, airfoil data was extrapolated to $\pm 180^{\circ}$, using Viterna's method. ${ }^{19}$ Finally, for each section, a two-dimensional cubic B-spline (the function "bisplrep" from FITPACK, also known as DIERCKX) was fitted to the lift and drag curves separately as functions of Reynolds number and angle of attack. A small amount of smoothing was used on each spline to reduce any high-frequency noise that could cause artificial multiple solutions ( 0.1 for lift, 0.001 for drag). Many BEM implementations use linear interpolation to estimate lift and drag coefficients; however, such an approach is unsuitable for gradient-based optimization as it introduces discontinuities in the derivatives.

\section{II.A.3. Rotor Aerodynamic Analysis}

The aerodynamic power predicted from the BEM method was modified to account for losses in the drivetrain. The drivetrain efficiency was assumed to vary with the aerodynamic power normalized by the rated power $\left(\bar{P}=P_{\text {aero }} / P_{\text {rated }}\right)$ as

$$
\eta_{d t}=1.0-(a / \bar{P}+b)
$$

where $a=0.0129$, and $b=0.0851$. This drivetrain efficiency curve is for a three-stage geared design and comes from an NREL study ${ }^{20}$ using WindPACT data. ${ }^{21}$ The net power produced is $P=\eta_{d t} P_{a e r o}$. Note that the maximum drivetrain efficiency is $91.5 \%$. Including this loss had a significant effect on total annual energy production and the estimated rated speed. Although this is less important in comparing relative aerodynamic performance between designs, it does have a significant effect on overall cost of energy.

The simulation methodology allows for any machine type combination of fixed/variable speed and fixed/variable pitch. However, all studies in this paper focus on variable-speed, variable-pitch machines. A typical power curve for a variable-speed, variable-pitch turbine is shown in Figure 3. Region 1 has no power generation as it occurs below the cut-in speed. In Region 2, variable-speed turbines operate at the optimal tip-speed ratio until either rated power or the maximum rotation speed is reached. Region 2.5 was added to handle the transition from Region 2 to Region 3. Rotor speed was fixed going into Region 2.5 and continuing into Region 3. Blade pitch was varied (pitch toward feather) in Region 2.5 and Region 3, so that torque varied smoothly in Region 2.5, and rated power was maintained in Region 3. The optimal tip-speed ratio for operation in Region 2 was determined externally as part of the optimization problem. 


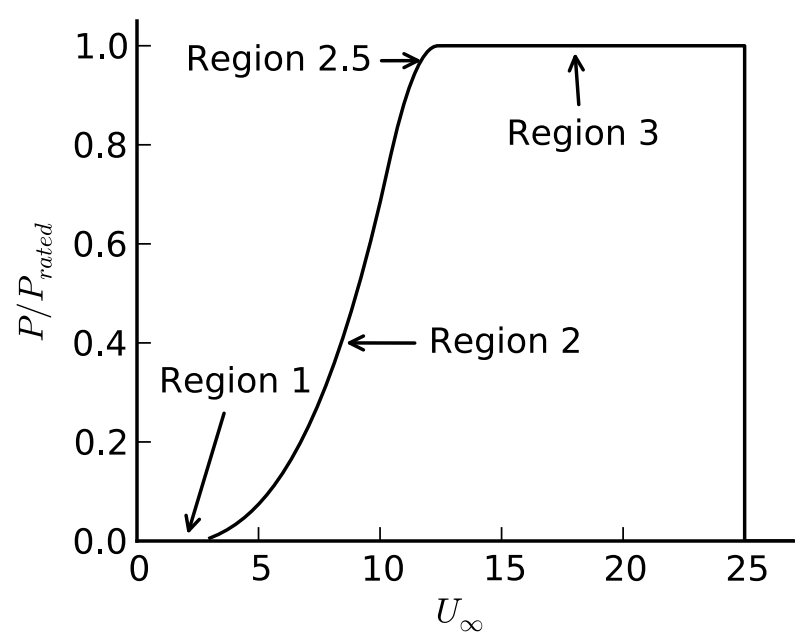

Figure 3. A typical power curve for a variable-speed, variable-pitch machine. Different regions of operation are shown.

AEP was computed using a Rayleigh distribution with a mean speed of $10 \mathrm{~m} / \mathrm{s}$, as specified in the International Electrotechnical Commission (IEC) standard for Class I turbines. ${ }^{22}$ Losses caused by wake interference from other turbines in the wind farm and losses caused by electrical grid unavailability were estimated simply using an array loss factor and an availability factor. These studies assumed that the grid was available $95 \%$ of the year, and that $10 \%$ of the potential wind farm AEP was lost because of wake interference effects.

\section{II.B. Rotor Structure}

\section{II.B.1. Beam Finite Element Analysis}

A beam finite element code, called pBEAM (polynomial beam element analysis module), was developed for the structural analysis. The methodology uses Euler-Bernoulli beam elements with 12 degrees of freedom (three translational and three rotational at each end of the element); the basic theory is described in any standard finite element textbook (see Yang ${ }^{23}$ ). A unique feature of the code is that section properties can be described as polynomials of any order between nodes. This means that, rather than using precomputed structural matrices for an assumed distribution and fixed shape-functions, the matrix coefficients are recomputed for each geometry. However, because the distribution is a polynomial, this can be done analytically using polynomial integration. For example, the bending stiffness can be described as varying quadratically across an element as $[E I](\eta)=[E I]_{2} \eta^{2}+[E I]_{1} \eta+[E I]_{0}$, where normalized coordinates $\eta$ are used across a given element. The bending stiffness matrix is then computed as

$$
K_{i j}=\frac{1}{L^{3}} \int_{0}^{1}[E I](\eta) f_{i}^{\prime \prime}(\eta) f_{j}^{\prime \prime}(\eta) d \eta
$$

where $f(\eta)$ are appropriate shape functions and $L$ is the length of the element. Other matrices (e.g., bending inertia matrix, incremental stiffness matrix, axial stiffness matrix, and axial inertia matrix) are computed similarly.

This approach allows for higher fidelity in describing variation in structural properties, which means that higher accuracy can be achieved with fewer elements (this is not particularly relevant for the rotor problem that typically must use a linear variation between section properties, but for other shapes like a cylindrical shell tower, where the moment of inertia varies quarticly, this variation is captured much more efficiently using this approach). The use of polynomials also allows for higher accuracy because integrals and derivatives are evaluated analytically rather than numerically. pBEAM can estimate structural mass, deflections in all degrees of freedom, coupled natural frequencies, critical global axial buckling loads, and axial stress. The code is written in $\mathrm{C}++$ and linked to the framework using Boost.Python.

\section{6 of 31}




\section{II.B.2. Sectional Composite Analysis}

An existing National Wind Technology Center code, PreComp, was used to estimate equivalent sectional inertial and stiffness properties of composite blades. ${ }^{24}$ PreComp uses modified classic laminate theory combined with a shear-flow approach. In addition to inertial and stiffness properties, its computation of the elastic center was used in this analysis. PreComp requires the geometric description of the blade (e.g., chord, twist, section profile shapes, and web locations), along with the internal structural layup (e.g., laminate schedule, orientation of fibers, and laminate material properties). It allows for high flexibility in the specification of the composite layup both spanwise and chordwise. An object-oriented description of the blade geometry and section composite layup in Python was linked to the Fortran code PreComp using f2py.

The finite element code pBEAM performs all computations about the elastic center of a structure and in principal axes to remove cross-coupling terms. Thus, the flapwise, edgewise, and coupled stiffness properties in the local blade coordinate system from PreComp are translated to the elastic center and rotated to principal axes, as described by Hansen. ${ }^{25}$ Similarly, input flapwise and edgewise loads are rotated to the principal axes, and output deflections are rotated back to the flapwise and edgewise axes.

Distributed aerodynamic blade loads were computed, as described previously. Weight loads were rotated from the inertial frame to the airfoil frame (through several intermediate frames). Because the analysis was done at the elastic center and in the principal axes, the strain was computed simply as

$$
\epsilon_{z z}(x, y)=\frac{M_{x}}{[E I]_{x}} y-\frac{M_{y}}{[E I]_{y}} x+\frac{N_{z}}{[E A]}
$$

For each airfoil section, the maximum strain location was assumed to occur in the outer skin layer of the chordwise location of maximum thickness.

For most of the analysis the strain was used directly, but for the fatigue analysis the stress was needed. To estimate the stress, a smeared effective modulus of elasticity was computed using classical laminate theory. For a given laminate stack, the constitutive equations are

$$
\left[\begin{array}{c}
N \\
\hdashline M
\end{array}\right]=\left[\begin{array}{c:c}
A & B \\
\hdashline B & D
\end{array}\right]\left[\begin{array}{c}
\epsilon^{0} \\
\hdashline k
\end{array}\right]
$$

where $\mathrm{N}$ and $\mathrm{M}$ are the average forces and moments of the laminate per unit length, and $\epsilon^{0}$ and $k$ are the mid-plane strains and curvature (see Halpin). ${ }^{26}$ If we denote the above matrix as $S$ and its inverse as $S^{*}$, then

$$
\epsilon_{z z} \approx S_{11}^{*} N_{z}
$$

This expression ignores laminate shear and bending moment effects (the latter would be zero for a symmetric laminate), which is a good approximation for slender turbine blades. At the same time, an effective smeared modulus of elasticity can be computed by integrating across the laminate stack

$$
E_{z z}=\frac{1}{\epsilon_{z z} h} \int_{-h / 2}^{h / 2} \sigma_{z z} d h=\frac{N_{z}}{\epsilon_{z z} h}
$$

where $N_{z}$ is the average force per unit length of the laminate. Combining equations (7) and (8) yields an estimate for the effective axial modulus of elasticity

$$
E_{z z}=\frac{1}{S_{11}^{*} h}
$$

Finally, the average stress in a laminate at a given section is given as

$$
\sigma_{z z}=E_{z z} \epsilon_{z z}
$$

\section{II.B.3. Additional Structural Considerations}

In addition to the capabilities of the finite element analysis, two additional structural considerations specific to the rotor problem were added. The first was a panel buckling calculation and the second was a fatigue cycle estimation. Both considerations can be particularly important for very large blades. ${ }^{27}$ This analysis 
considered only spar-cap buckling; however, detailed design should also consider trailing-edge panel buckling. The panel buckling calculation uses a relatively simple method described by Bir. ${ }^{28}$ The calculation results from an eigenanalysis of a flat panel that is loaded edgewise. The derivation assumes that the width of the panel is much smaller than the length of the panel, which is true for the rotor blades (except near the tip). It is also assumed conservatively that the longitudinal edges are simply supported, while the chordwise edges are free. Under these assumptions, the critical edge load per unit length is given by

$$
N_{c r}=3.6(\pi / b)^{2} D_{11}
$$

where the $D$ matrix is the same as in Eq. (6) and corresponds to that for the laminate stack of the spar cap on the surface in compression. The length scale $b$ is the width of the spar cap. The critical buckling strain for the section is then given by

$$
\epsilon_{c r}=\frac{-N_{c r}}{h E_{z z}}
$$

where $h$ is the thickness of the spar cap, $E_{z z}$ is computed from Eq. (9), and the negative sign is due to the fact that the buckling loads are compressive.

Finally, a fatigue calculation for the blade root was included. A full lifetime fatigue analysis can be quite complex; however, a simplistic assessment can be done using the edgewise gravity loads, as these loads fully reverse every blade rotation. Although edgewise gravity loads are not always the dominant load condition, they can be more significant than flapwise aerodynamic loads in determining the fatigue strength of very large blades. ${ }^{27}$ This constraint is not a replacement for a full fatigue analysis, but was used to prevent unrealistically small chord sizes at the blade root. The blade root uses thick laminates to stiffen the connection to the pitch bearing, and without a proper constraint provides a large incentive for the optimizer to decrease the chord at the blade root.

To aid the fatigue estimation, it is assumed that the $\mathrm{S}-\mathrm{N}$ curve for the root section can be parameterized as

$$
S_{f}=a N^{b}
$$

where $\mathrm{b}$ is assumed to be -10 (a typical value for glass-reinforced composite materials). ${ }^{29}$ The maximum stress at the root of the blade caused by only the gravity loads was computed as described above. Because this loading is fully reversed, the stress value at the 3 o'clock azimuth can be used directly as the damage equivalent load for the S-N curve. An average rotation speed was estimated by computing the expected value of the rotor speed using the wind speed distribution

$$
\bar{\Omega}=\int_{V_{\text {in }}}^{V_{\text {out }}} \Omega(V) f(V) d V
$$

where $f(V)$ is a probability distribution function of the wind speeds (a Rayleigh distribution with a mean wind speed of $\bar{U}=10.0 \mathrm{~m} / \mathrm{s}$ was used, as discussed previously). A 20-year lifetime of continuous rotation was assumed. The value for $a$ in Eq. (13) was calibrated using the loading conditions for the baseline rotor so that, at 20 years, the root stress had a $10 \%$ margin relative to the fatigue stress (i.e., $\sigma_{\text {root }} / S_{f}=0.9$ ). The margin was added to avoid overly constraining the problem because it was only defined relative to the baseline. For a new design, the maximum fatigue stress for the number of cycles was required to be less than the fully reversed stress at the blade root

$$
\left|\sigma_{\text {root-gravity }}\right|<S_{f}
$$

where the stress was due only to gravity loads and was computed at the blade root.

\section{II.C. Cost Model}

The cost model was based primarily on the NREL cost and scaling model,${ }^{10}$ with a few modifications that were found to be important for this study. First, the rotor mass was computed from the structural model instead of the scaling law. Blade cost was a linear function of blade mass, with coefficients derived from an internal NREL study. ${ }^{30}$

Next, the tower mass estimation was replaced. The current cost and scaling relationship scales linearly with the square of the rotor diameter (for a fixed hub height). Although this is reasonable under the 
assumption that the entire turbine scales proportionally, it was not reasonable for many of the studies in this paper. For example, some studies examined varying the rotor diameter at a fixed power rating. At a fixed rating, the thrust loads and rotor mass change relatively slowly with increased rotor diameter. The scaling law, however, predicts a quadratic variation with diameter, which greatly overestimates the impact on the tower mass. Even worse, other studies in this paper varied the rated power at fixed rotor diameter. In this case, the cost and scaling relationship would predict no change in tower mass, because the diameter is fixed, but in reality the thrust loads would increase significantly. Because tower mass is such a large fraction of the total turbine mass, it is important to capture changes in tower mass in a more realistic manner. Although capturing these changes does not affect the conclusions of this paper (as we only compared the relative value of different objectives), obtaining a more realistic tower mass estimate is desirable to produce a more accurate estimate of the various metrics.

A full tower model was developed for this study. However, as stated previously, the scope of this paper was intentionally limited to only include rotor design variables. Thus, a simpler scaling relationship for tower sizing was desired, and derived in the following calculations. For a tapered tower, the critical stress location is somewhere in the middle (the bending moment is largest at the base, but so is the moment of inertia). For simplicity, we assumed that the critical location was halfway up the tower. Then, the stress at that location, which reaches some critical value for either ultimate stress or buckling, is

$$
\sigma_{c r}=\frac{\left(m_{R N A}+m_{\text {tower }} / 2\right) g}{A_{\text {mid }}}+\frac{T H r_{\text {mid }}}{2 I_{\text {mid }}}
$$

where $\mathrm{r}$ is the radius of tower (for simplicity we assume half of the tower mass is above that location). For a cylindrical shell section, the moment of inertia is proportional to the area

$$
I=\frac{A r^{2}}{2}
$$

Substituting this in for $I_{m i d}$ and solving in terms of cross section area gives

$$
A_{\text {mid }}=\frac{1}{\sigma_{c r}}\left[\left(m_{R N A}+m_{\text {tower }} / 2\right) g+\frac{T H}{r_{\text {mid }}}\right]
$$

The mass of the tower can then be estimated as

$$
m_{\text {tower }}=\rho \int_{0}^{H} A(z) d z
$$

If we assume that the tower resizes in such a way that the diameter-to-thickness ratio is constant, and the taper ratio of the tower is constant, then the mass of the tower can be directly related to its area at the midpoint

$$
m_{\text {tower }}=\rho \alpha A_{\text {mid }}
$$

where $\alpha$ is purely a function of the tower taper ratio (a constant). Because $A_{m i d}$ is a function of the tower mass, we can now solve directly for the tower mass. The resulting equation is

$$
m_{\text {tower }}=k\left[m_{R N A} g+\frac{T H}{r_{\text {mid }}}\right]
$$

where $k$ is a constant that is a function of the acceleration of gravity, the tower taper ratio, the tower effective material density, and the critical stress at the tower midpoint. Finally, if we assume that resized towers will have a similar stress utilization at the midpoint, then the mass of the tower should scale as

$$
\frac{m_{\text {tower }}}{m_{\text {tower } 0}}=\frac{\left[m_{R N A} g+T H / r_{\text {mid }}\right]}{\left[m_{R N A} g+T H / r_{\text {mid }}\right]_{0}}
$$

Although this is not a universal scaling law, it should work well for cylindrical shell designs that are scaled relative to a baseline design at a constant taper ratio and constant diameter-to-thickness ratio. Strictly speaking, $r_{m i d}$ is a function of the tower mass given the sizing assumptions listed above. However, including that variation creates much more complexity in the mathematical expression and has little effect as the 
change in radius is relatively small. For simplicity we kept it fixed at the radius of the reference design $\left(r_{\text {mid }}=2.4675\right)$.

This sizing works well for increasing tower mass; however, it cannot be expected to continue indefinitely for shrinking tower masses. Other constraints, such as buckling, natural frequency, and deflection will become critical and constrain the tower from shrinking further. Without implementing a full tower model, we can estimate how these constraints will affect the tower sizing. For a fixed top mass and tower height, and by neglecting the smaller effect of the distributed tower mass, the natural frequency of the tower is proportional to $I^{0.5}$. Typically, the tower is constrained by the $1 P$ frequency of the rotor, as is the case with the 5-MW reference model, so the moment of inertia can not shrink too far before this becomes a critical constraint. Similarly, for a given thrust and tower height, the deflection of the tower top scales with $I^{-1}$. Again, smaller moments of inertia increase the tower top deflection, which will cause this constraint to become critical. If we use the formula for column buckling and consider only the stress from the thrust for simplicity, then the buckling criteria $\sigma / \sigma_{c r}$ is proportional to $I^{-1.25}$. Clearly, shrinking the moment of inertia also decreases the buckling margin. In all of these constraints, the moment of inertia is a crucial driver. As a surrogate for these constraints, we assumed that the moment of inertia of the tower cannot shrink more than $10 \%$ relative to the reference design

$$
\frac{I}{I_{0}}>0.9
$$

For a cylindrical shell tower with a fixed diameter-to-thickness ratio, the moment of inertia is proportional to $d^{4}$. Also, as shown previously, the tower mass is proportional to $d^{2}$ (for a fixed diameter-to-thickness ratio and fixed taper ratio). Thus, for these assumptions, the tower mass is proportional to $I^{0.5}$, and the constraint on the moment of inertia becomes

$$
\frac{m_{\text {tower }}}{m_{\text {tower } 0}}>\sqrt{0.9}
$$

Therefore, the tower mass is estimated as

$$
\frac{m_{\text {tower }}}{m_{\text {tower } 0}}=\max \left(\sqrt{0.9}, \frac{\left[m_{R N A} g+T H / r_{\text {mid }}\right]}{\left[m_{R N A} g+T H / r_{\text {mid }}\right]_{0}}\right)
$$

This function max introduces a discontinuity in its derivative, and so it cannot be directly used with gradientbased optimization. Instead, a small cubic spline is added to provide a smooth transition between the two tower mass regions.

The tower cost estimate was unmodified from the simple linear scaling of the NREL cost and scaling model. Hub mass and cost and nacelle mass and cost were also unmodified. The nacelle calculation already scales with relevant variables for this study, such as the hub thrust, torque, and maximum rotor rotation speed. Improved drivetrain/nacelle models were developed, but for the scope of this paper, the simpler scaling relationships used in the cost and scaling model were sufficient.

Finally, a modified balance-of-station (BOS) cost model was used. NREL recently developed a new BOS model for land-based wind turbines. ${ }^{31}$ The previous BOS model scales simply based on machine rating, and does not accurately scale up to the large machine ratings of today. The new version uses a substantially different bottom-up approach by estimating component quantities and sizes. Operations and maintenance costs remain unchanged from the NREL cost and scaling model.

\section{II.D. Reference Geometry}

The reference geometry used in this study was based on the NREL 5-MW reference wind turbine. ${ }^{32}$ The airfoils and chord schedule used in the development of the blade model were adopted from the Dutch Offshore Wind Energy Convertor project. ${ }^{33,34}$ Aerodynamic properties were specified in terms of airfoil polar curves which spanned the full angle of attack range. Structural stiffness was given in terms of axial, flapwise, and edgewise stiffness. Inertia characteristics were expressed in terms of mass per unit length, and flapwise and edgewise area moments of inertia. To arrive at a description of the structural stress/strain field under different loading conditions, a composite-material lay-up was also defined at 38 span sections. A preliminary version of the initial layup was provided by Sandia National Laboratories, ${ }^{35}$ as a NuMAD ${ }^{36}$ property file. The layup was constructed so as to reproduce the prescribed stiffness/inertia properties along the blade span as close as possible while still satisfying structural constraints. Material properties were largely taken from the U.S. Department of Energy (DOE)/Montana State University (MSU) Composite Material Fatigue 
Database $^{29}$ and a Sandia large rotor study, ${ }^{27}$ but were modified somewhat to represent generic prestacked laminates. The various materials included: GelCoat, glass fabrics (Unidirectional (E-LT-5500, [0]2), SNL TRIAX ([ \pm 45$] 2[0] 2)$, SaerTex Double-Dias (DB, $[ \pm 45] 4)$, carbon fabrics (generic unidirectional with an effective thickness of $0.47 \mathrm{~mm}$ ), generic foam, and epoxy resins. The structural layup was not intended to be a fully assessed blade design, but a good starting point for comparative studies (such as the study described in this paper). Unidirectional carbon was chosen to both reduce the tip deflection and potential tower strike, and to match the stiffness distribution of the original NREL 5-MW model.

Starting from the NREL 5-MW reference design, a parameterization was needed for subsequent optimization. Ideally, the rotor geometry should be parameterized with a small number of terms for optimization efficiency, yet still allow for significant flexibility in describing the geometry. The chord was parameterized with five variables, shown in Figure 4a. The first and last chord positions were fixed at the root and tip, respectively. The third chord variable was fixed at $62.6 \%$ of the blade length. The radial location of the second chord variable (typically the location of the maximum chord) was itself a variable $\left(r_{2}\right)$. An Akima spline $^{37}$ was fit to the four radial/chord pairs to compute the chord at any other radial station ${ }^{\mathrm{a}}$. The airfoil shapes were fixed, therefore, changes in chord were directly proportional to changes in airfoil thickness (fixed $t / c$ for a given section).

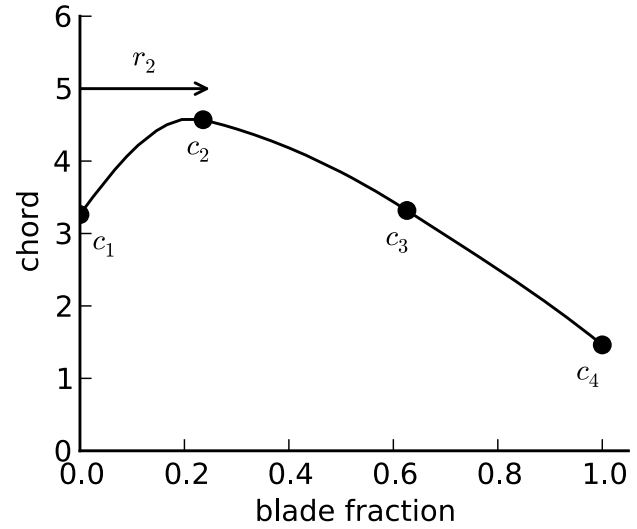

(a) Chord distribution parameterization.

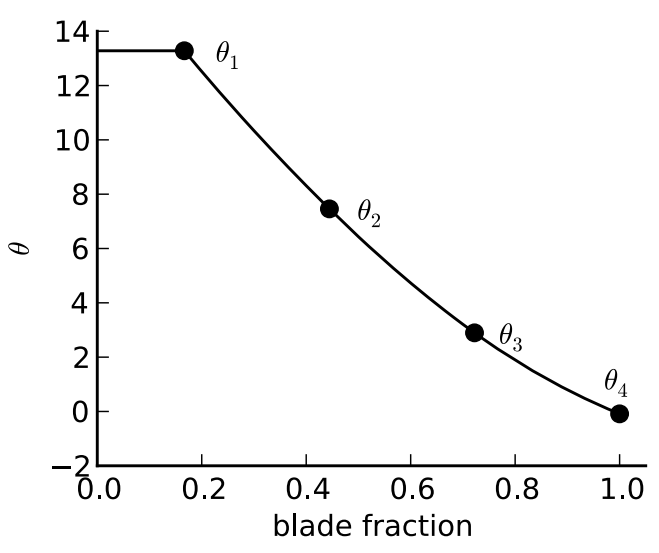

(b) Twist distribution parameterization.

Figure 4. Parameterization of rotor blade chord and twist distribution.

The twist distribution was parameterized in a similar manner. The inboard portion of the blades has cylindrical sections, which are invariant to twist. Thus, a constant value was used - up to $16.7 \%$ of the blade length where the first airfoil was defined. Twist was defined at four linearly spaced radial points from this point to the blade tip, and was fitted with an Akima spline.

This parameterization led to a baseline model that was not exactly the same as the NREL 5-MW reference model. For both the chord and twist distribution, parameters were chosen to best fit the reference model's chord and twist (the interpolation was done along the radial stations defined for the structure, as opposed to the aerodynamics, simply because more chord/twist points were defined for the structure). The fit was found by solving the optimization problem

$$
\begin{array}{ll}
\operatorname{minimize} & \left.\| \operatorname{chord}_{\mathrm{NREL}}-\operatorname{chord}_{\text {Akima }}(x)\right) \|_{2}^{2} \\
\text { with respect to } & x=\left\{r_{2}, c_{1}, c_{2}, c_{3}, c_{4}\right\}
\end{array}
$$

with a corresponding minimization problem for the twist. The chord and twist distributions for the NREL 5-MW reference model are compared to the best fit designs in Figure 5. The chord distribution is very similar except near the tip. The twist distribution matches almost exactly.

\footnotetext{
${ }^{a}$ An Akima spline was chosen because of its robustness to outliers. If one of the chord variables differs significantly in magnitude from the others (which can happen during the course of an optimization), then oscillations are produced for many spline types. This may cause some sections to have negative chord values, which is nonphysical and will prevent the analysis from running properly. An Akima spline prevents these types of oscillations. A simple bound constraint on chord is sufficient to prevent intermediate designs with negative chord, as opposed to a nonlinear constraint on chord that would be required if using a cubic spline or Bezier curve.
} 


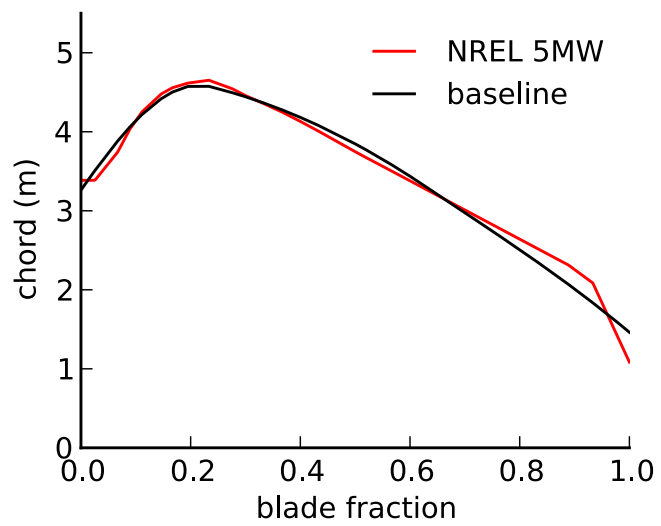

(a) Comparison of chord distribution.

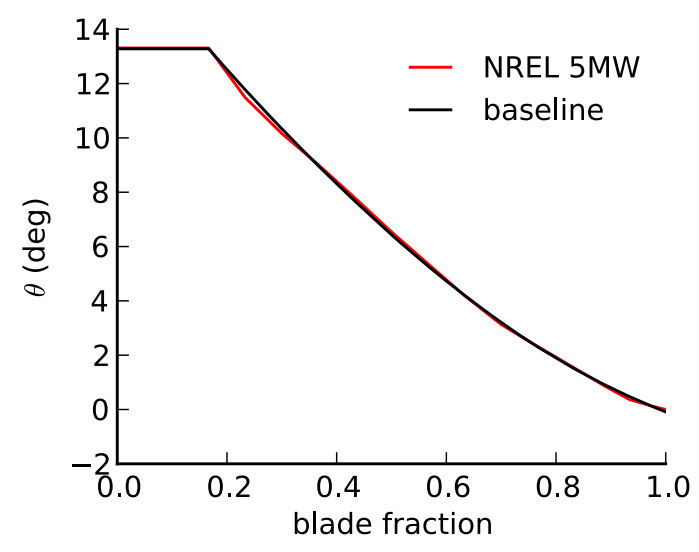

(b) Comparison of twist distribution.

Figure 5. Comparison between the NREL 5-MW reference model and the parameterized baseline model derived as a best fit to the NREL model.

For the reference model, the spar cap was significantly thicker over the first half-meter. Because of the abrupt change, rather than attempting to fit a spline over the entire span, the spar cap thickness over the first half-meter of the blade length was assumed fixed. For simplicity, the spar cap thickness over the rest of the cylindrical section was assumed constant but not fixed (from $r=2$ to $r=11.75$ - note that these locations were normalized by the blade length to accommodate resizing of rotor diameter). Over the outer portion, spar cap thickness was parameterized in the exact same way as the twist distribution (defined at four linearly spaced stations and fitted with an Akima spline), except for the spar cap thickness at the blade tip, which was fixed to prevent unrealistically small thicknesses. The parameterization of the spar cap thickness is shown in Figure 6.

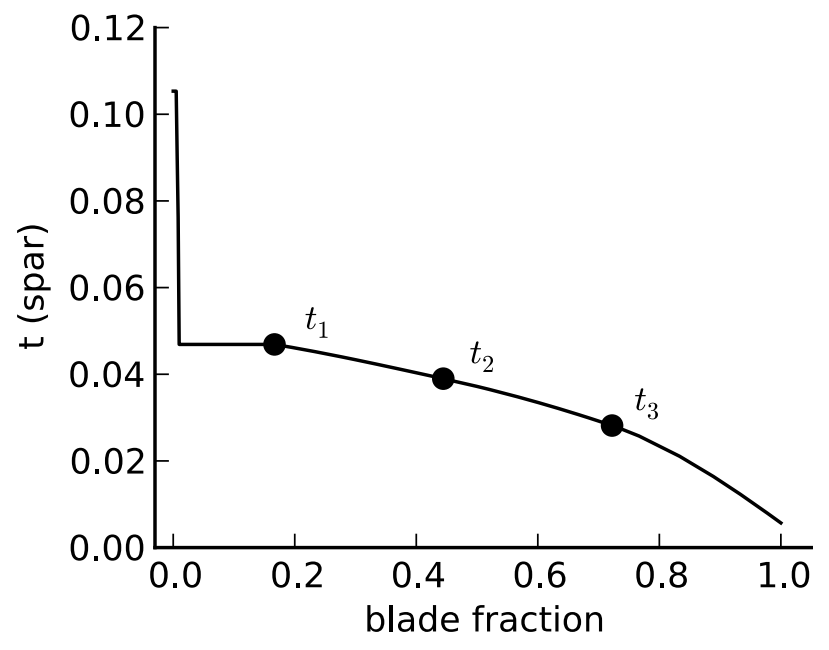

Figure 6. Parameterization of spar cap thickness distribution.

The baseline spar cap thickness was not resized using a best fit to the reference model. Because a preliminary version of the 5-MW reference structural layup was used, the structure was found to buckle at the extreme load case. Though the buckling model used in this analysis was simplistic, the results were consistent with a linear-buckling analysis conducted in ANSYS, which also predicted buckling in the spar cap and trailing-edge panels. Using an infeasible design as the baseline would lead to rather unfair comparisons. Instead, the spar cap was resized to satisfy the buckling constraint everywhere along the span. 
The optimization problem was defined as

$$
\begin{array}{ll}
\operatorname{minimize} & m_{\text {rotor }}(x) \\
\text { with respect to } & x=\left\{t_{1}, t_{2}, t_{3}\right\} \\
\text { subject to } & b m(x)_{j}>0, j=1, \ldots, n
\end{array}
$$

where $b m$ is the buckling margin computed at every section in the structure at the extreme load condition (see Section II.E for a description of the buckling margin calculation - note that a slightly larger safety factor $\gamma_{f}=1.36$ was used to ensure that the baseline design was strictly feasible).

The new spar cap thickness distribution was compared with the original structural layup for the NREL 5-MW reference model in Figure 7a. The thickness was larger toward the root and tip to satisfy the buckling constraints. The strain on the blade's upper and lower surface at the extreme load and the corresponding buckling limit is shown in Figure 7b. Compared to the original, the modifications caused the mass to increase by $5 \%$. For the remainder of the paper, all reference quantities refer to the modified baseline model, as opposed to the original NREL 5-MW reference model. Some of the relevant reference quantities for the baseline model are defined in Table 1. Comparisons of the mass distribution, flapwise stiffness, and edgewise stiffness with the original NREL 5-MW model are shown in Figure 8.

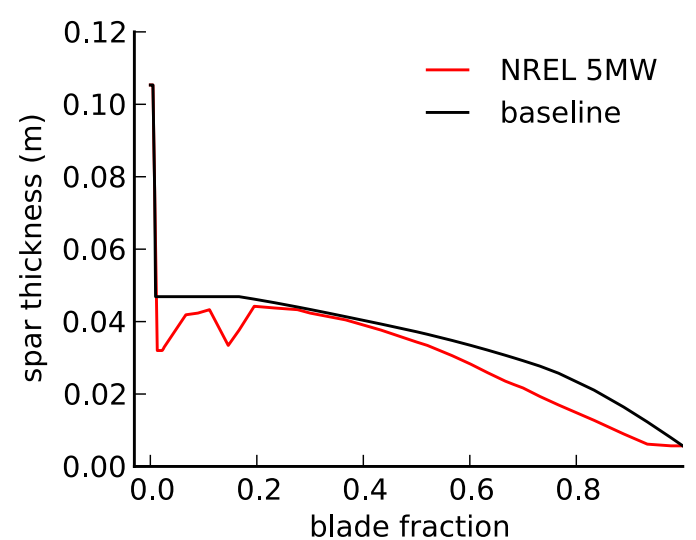

(a) Comparison between NREL reference design and the resized baseline model.

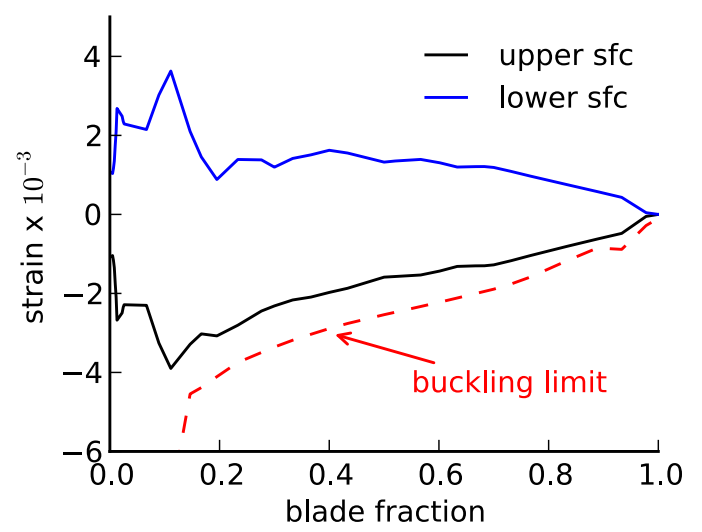

(b) Strain on upper and lower surface ("sfc" in figure) of the baseline design and the buckling limit.

Figure 7. Spar cap thickness distribution was sized to minimize mass and satisfy the buckling constraint at the extreme load condition.

Table 1. Various normalization and reference quantities for the baseline design.

\begin{tabular}{ll}
\hline AEP & $20.6 \times 10^{6} \mathrm{kWh}$ \\
blade mass & $18,246 \mathrm{~kg}$ \\
turbine mass & $657,180 \mathrm{~kg}$ \\
tip deflection (at rated speed) & $2.44 \mathrm{~m}$ \\
cost of energy & $0.0498 \$ / \mathrm{kWh}$ \\
\hline
\end{tabular}




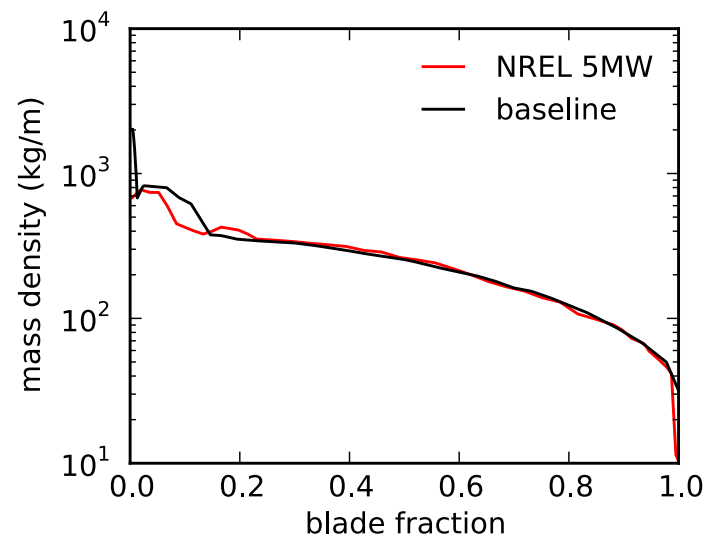

(a) Mass density distribution.

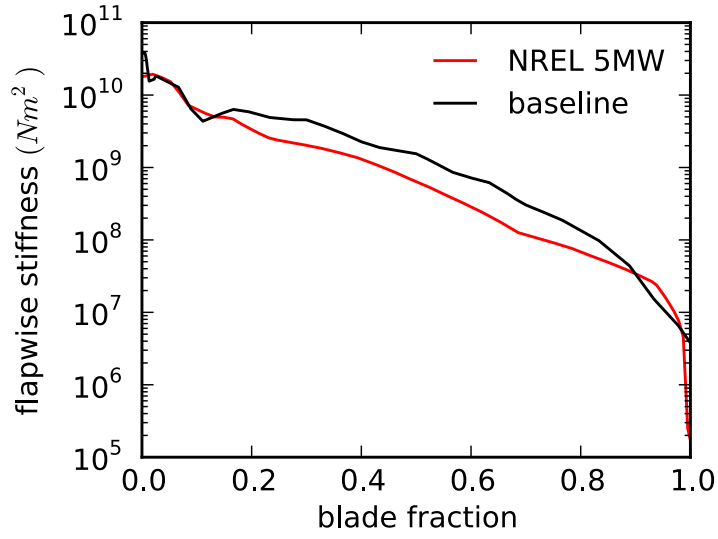

(b) Flapwise stiffness distribution.

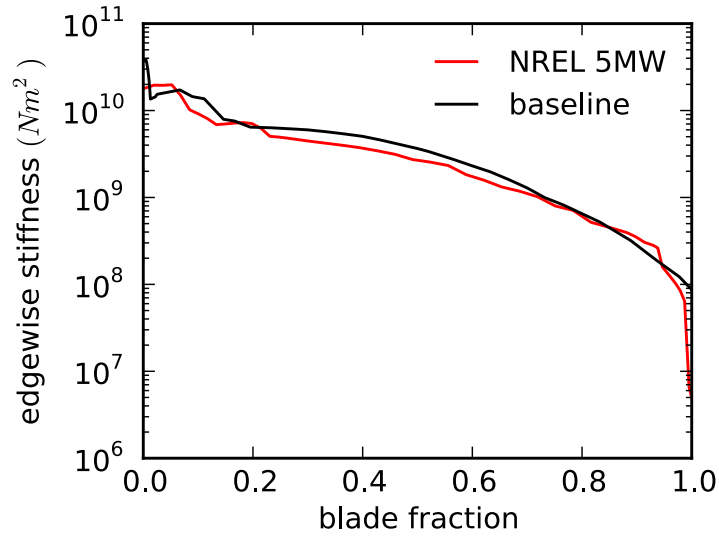

(c) Edgewise stiffness distribution.

Figure 8. Comparison between some of the structural properties of the NREL 5-MW reference design and the baseline design. The baseline design was derived from Sandia's layup schedule, but was modified slightly to fit the geometry parameterization and prevent buckling.

\section{II.E. Optimization Strategy and Constraints}

Several optimizers, both commercial and open source, were tested on the optimization problems described in this paper. The active-set algorithm of MATLAB's function "fmincon", which uses a sequential quadratic programming method, was found to be the most robust for these particular problems and was used for all reported results. Because none of the analysis code was implemented in MATLAB, there was a performance penalty due to having to reinstantiate all objects at every iteration. However, the robustness of the algorithm was more important for this study than the small loss in computational speed.

Gradients were estimated using central differencing, and a multistart approach was used to increase the likelihood of finding the global optimum. Early on, forward differencing was used to estimate the gradients, and the multistart approach was found to be necessary, as some starting points terminated prematurely. However, the better gradient estimates provided by central differencing resulted in improved convergence behavior, and even with multiple starting points, the same optimal solution was always found. This suggests that the best solution was found for each study. On the other hand, use of alternative optimizers may require more accurate gradients that are produced by other means, such as the complex-step method ${ }^{38}$ or hyper-dual numbers. ${ }^{39}$

All objectives and constraints were normalized, so that they were of order one for improved scaling. For example, an objective minimizing the ratio of turbine mass to AEP is implemented as

$$
\min \frac{m / m_{0}}{A E P / A E P_{0}}
$$

In the following optimization problems, these normalization constants are not explicitly written to reduce 
clutter; the objective is simply denoted as minimum $m / A E P$. All solutions were converged to a function tolerance of $1 \times 10^{-6}$ and a constraint tolerance of $1 \times 10^{-5}$. Bound constraints were set large enough to never be active, unless otherwise noted.

A real turbine must be designed to meet a very large number of structural constraints. ${ }^{22}$ Only a handful of representative cases were used for this purpose. First, an ultimate strength analysis was performed at an extreme load condition. The 50-year extreme wind condition is defined as $V_{e 50}=1.4 V_{\text {ref }}\left(V_{\text {ref }}=50\right.$ $\mathrm{m} / \mathrm{s}$ for class I turbines). ${ }^{22}$ The distributed weight loads were added to the aerodynamic loads at 0 degrees pitch and the 3 o'clock azimuthal position, which is the worst case for the edgewise loads. Flapwise and edgewise aerodynamic and structural loads at $V_{e 50}$ are shown in Figure 9. The flapwise loads are primarily aerodynamic and the edgewise loads are primarily due to the weight, but not entirely. The corresponding strain distribution is shown in Figure $7 \mathrm{~b}$.

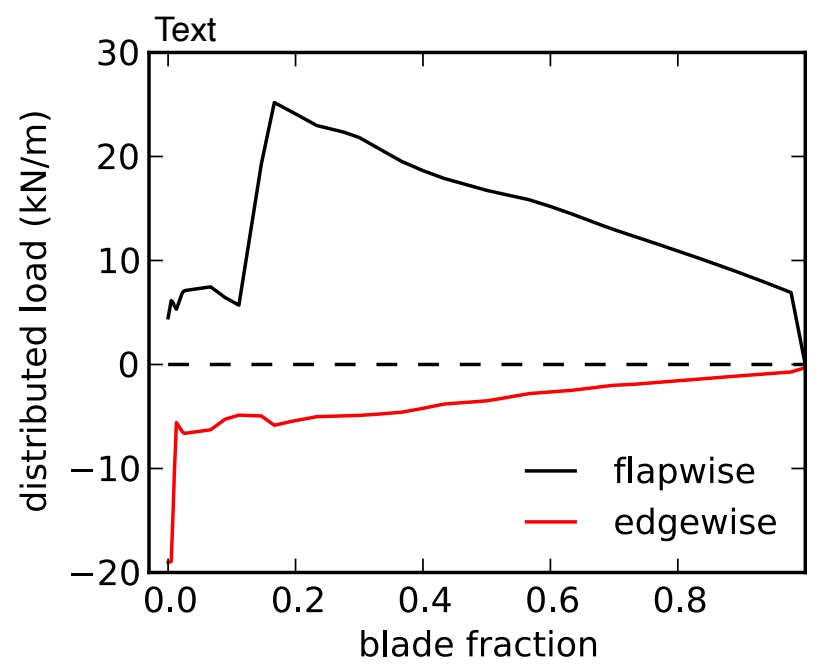

Figure 9. Flapwise and edgewise loading along the blade at the 50-year extreme load condition.

A maximum strain condition was used

$$
\epsilon_{50} \leq \frac{1}{\gamma_{f} \gamma_{m}} \epsilon_{u l t}
$$

where the partial safety factor for loads $\left(\gamma_{f}\right)$ was set at 1.35 , and the partial safety factor for materials $\left(\gamma_{m}\right)$ was set at 1.1 per the IEC requirements. ${ }^{22}$ (For actual designs the material and loading partial safety factors are more involved than using a simple number, and should include additional knockdowns for uncertainty in material properties, expected environmental conditions, etc. For the purposes of this paper, this additional complexity is unnecessary.) Because the spar cap is primarily carbon, representative numbers of ultimate strain in tension and compression are about $2 \%$ and $1 \%$, respectively. Conservatively, the smaller value of $1 \%$ was used as the ultimate strain $\left(\epsilon_{u l t}\right)$. Only the strain from a few representative sections was used to reduce the number of constraints. The sections were biased inboard as those were the critical locations. The sections were at $0 \%, 11.1 \%, 30 \%$, and $63.3 \%$ of the blade length on both the upper and lower surface. Although using a large number of constraints is not problematic, it is also not necessary because the chord and thickness must vary smoothly due to the parameterization, which leads to relatively smooth variations in the strain.

Panel buckling was estimated using the simplified method described in section II.B.3. The buckling margin was computed as

$$
b m=\epsilon_{50} \gamma_{f}-\epsilon_{c r}
$$

where both values are negative because the structure is in compression for a buckling condition. The corresponding constraint was

$$
\frac{b m}{\epsilon_{u l t}}>0
$$


where the ultimate strain was only used so that the resulting constraint was better scaled. Similar to the strain case, only a few representative stations were used. These stations were at $16.7 \%, 36.7 \%, 63.3 \%$, and $83.3 \%$ of the blade length on the upper surface only.

Although the constraints on extreme loading are useful when sizing the structure, without additional constraints the optimum structures would be far too flexible to be practical. As a result, constraints on the maximum tip deflection and natural frequency of the blade were added to ensure adequate stiffness. The deflection of the structure was computed at rated speed in the 3 o'clock azimuth position (worst case). Because conceptual rotor design does not often have a defined substructure model, we could not use bladestrike as the constraint criteria. Instead, we assumed that the baseline rotor was designed with adequate stiffness and therefore constrained the deflection to be within $10 \%$ of the baseline tip deflection.

$$
\delta<1.1 \delta_{0}
$$

The other constraint related to blade stiffness was on the natural frequencies of the blades. To avoid structural resonance issues, the first natural frequency (and thus all natural frequencies) of the blade should be above the maximum rotor blade passing frequency

$$
\omega_{1}>\gamma_{\text {freq }}\left(3 \Omega_{\text {rated }}\right)
$$

where the safety factor $\gamma_{f r e q}$ was set to be 1.1. The maximum rotation speed occurs at rated speed, and the factor of 3 came from the number of blades.

The fatigue strength at the root was computed for a 20-year lifetime, as discussed in Section II.B.3. The fatigue constraint was imposed as

$$
-S_{f}<\sigma_{\text {root-gravity }}<S_{f}
$$

Finally, a constraint on the maximum tip speed was imposed as a surrogate for a noise constraint. This constraint was not imposed by the optimizer, but was implemented directly into the analysis, as described in Section II.A.3.

The nominal optimization problem is outlined below:

$$
\begin{array}{clr}
\underset{x}{\operatorname{minimize}} & J(x) & \\
\text { subject to } & \left(\gamma_{f} \gamma_{m} \epsilon_{50 i}\right) / \epsilon_{u l t}<1, i=1, \ldots, N & \text { ultimate tensile strain } \\
& \left(\gamma_{f} \gamma_{m} \epsilon_{50 i}\right) / \epsilon_{u l t}>-1, i=1, \ldots, N & \text { ultimate compressive strat } \\
& \left(\epsilon_{50 j} \gamma_{f}-\epsilon_{c r}\right) / \epsilon_{u l t}>0, j=1, \ldots, M & \text { spar cap buckling } \\
& \delta / \delta_{0}<1.1 & \text { tip deflection at rated } \\
& \omega_{1} /\left(3 \Omega_{\text {rated }}\right)>1.1 & \text { blade natural frequenc } \\
& \sigma_{\text {root-gravity }} / S_{f}<1 & \text { fatigue at blade root d } \\
& \sigma_{\text {root-gravity }} / S_{f}>-1 & \text { fatigue at blade root d } \\
& V_{\text {tip }}<V_{t i p_{\max }} & \text { maximum tip speed (in }
\end{array}
$$$$
\left(\gamma_{f} \gamma_{m} \epsilon_{50 i}\right) / \epsilon_{u l t}>-1, i=1, \ldots, N \quad \text { ultimate compressive strain }
$$$$
\delta / \delta_{0}<1.1 \quad \text { tip deflection at rated speed }
$$$$
\text { blade natural frequency }
$$$$
\text { fatigue at blade root due to gravity loads }
$$$$
\text { fatigue at blade root due to gravity loads }
$$$$
\text { maximum tip speed (imposed directly in the analysis) }
$$

Because this set of constraints is repeatedly in subsequent optimization problems, it will be referred to as $c_{\text {set }}(x)$, where the constraints are reorganized as needed so that feasibility occurs when $c_{\text {set }}(x)<0$. Design variables are summarized in Table 2. Not all design variables were used in every problem. As stated earlier, airfoil thickness was changed through the chord distribution, as the nondimensional airfoil shapes were fixed. 
Table 2. Design variables used in the optimization problems.

\begin{tabular}{lcc}
\hline description & variable name & number of parameters \\
\hline Chord distribution (Fig. 4a) & $\{c\}$ & 5 \\
Twist distribution (Fig. 4b) & $\{\theta\}$ & 4 \\
Spar cap thickness distribution (Fig. 6) & $\{t\}$ & 3 \\
Tip-speed ratio in Region 2 (Fig. 3) & $\lambda$ & 1 \\
Rotor diameter & $D$ & 1 \\
Machine rating & rating & 1 \\
\hline
\end{tabular}

\section{Results}

These studies examine the impact of using the following different objectives to optimize a wind turbine rotor: maximizing annual energy production (AEP), minimizing the ratio of turbine mass to AEP, and minimizing cost of energy. Below, fundamental differences and important considerations of each case are highlighted and values are reported as normalized by the reference design, where applicable (e.g., $\overline{A E P} \equiv$ $\left.A E P / A E P_{0}\right)$.

\section{III.A. Maximum Annual Energy Production}

Although typically the most appropriate metric for wind turbine optimization is minimizing the cost of energy (COE), many studies focus on optimizing the aerodynamic performance of a wind turbine rotor either by maximizing power at a fixed speed, or maximizing AEP for a given wind distribution. Pure aerodynamic optimization may be done for a variety of reasons, such as: an appropriate structural model is not available to the designer, an appropriate cost model is not available, the organization separates aerodynamic and structural design, or high-fidelity tools are used and, for computational efficiency, the aerodynamic and structural optimizations are decoupled. This section examines when pure aerodynamic optimization might be a suitable practice. In other words, under what circumstances does maximizing AEP, or sequentially maximizing AEP and then minimizing mass, yield a good design? A "good design" in this study means that it achieves a similar cost of energy as the minimum COE design.

Unfortunately, maximizing AEP without at least some consideration of the rotor mass is not a particularly well-defined problem. Part of the difficulty with using maximum AEP as an objective is that a multitude of solutions exist that produce essentially the same AEP (in other words, many local optima exist). To help clarify, consider a related optimization problem

$$
\begin{array}{ll}
\text { maximize } & A E P(x) \\
\text { with respect to } & x=\{\{c\},\{\theta\}, \lambda\} \\
\text { subject to } & c_{\text {set }}<0 \\
& \overline{m_{\text {blade }}}=m_{c}
\end{array}
$$

where $m_{c}$ is a constant mass value. The result of repeating this optimization problem at several discrete constraints on the blade mass $m_{c}$ is shown in Figure 10. Essentially the same AEP can be achieved across a wide range of designs with very different masses. Point M1 is noted on the figure as the design with fixed blade mass relative to the baseline design. Clearly, point M1 is superior to all points to the right of it, as it achieves essentially the same AEP, but with significantly reduced mass.

The chord and twist distributions of design M1 are compared with those of the baseline design and the Betz solution including tip losses ${ }^{40}$ (Figure 11). As expected, the tip-speed ratio increased, causing the solidity of the outer sections - where most of the power is produced - to decrease. At the same time, the chord distribution inboard increased, thus structural constraints like deflection and stress did not become critical. The outer portion of the optimized design is essentially a best fit to the Betz design, given the limited degrees of freedom. Note that the Betz distribution should not be directly compared to the baseline design, as design M1 operates at a higher tip-speed ratio (7.68 versus 7.55).

Although design M1 is superior to other designs in Figure 10 with the same AEP, we presume that structural calculations are not available to a designer attempting to maximize AEP (otherwise a different 


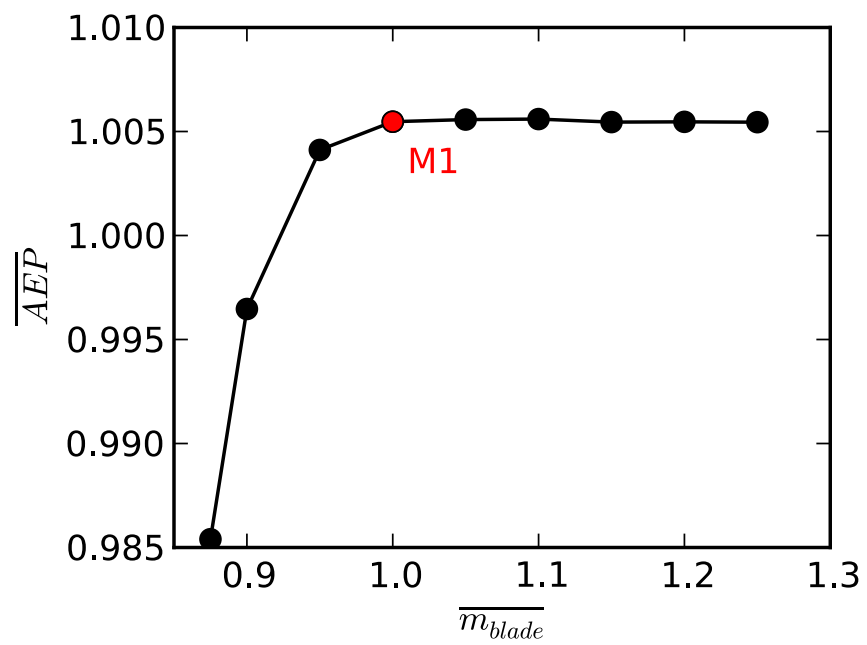

Figure 10. Maximum annual energy production for different designs. Each design was constrained to have a different blade mass. Point M1 is highlighted and corresponds to the maximum AEP solution, with the mass constrained so that $\overline{m_{\text {blade }}}=1$.

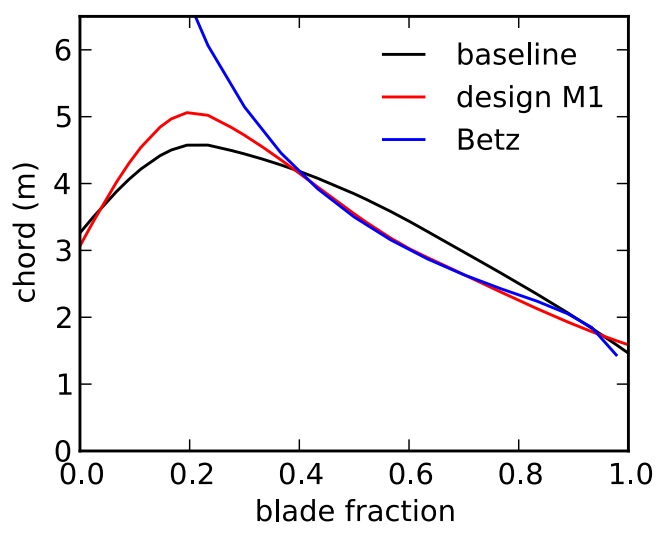

(a) Chord distribution along blade.

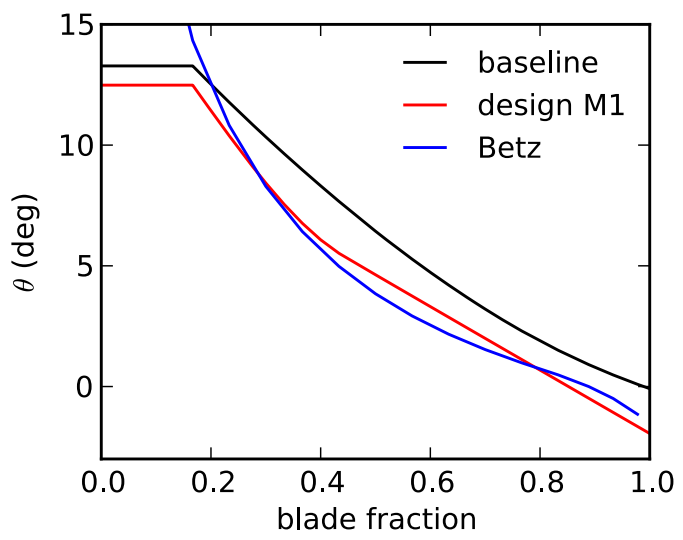

(b) Twist distribution along blade.

Figure 11. Chord and twist distribution for the maximum AEP design with fixed mass (design M1). Comparison is made to the baseline design and the Betz distribution for the tip-speed ratio of design M1. 
objective would be used). Clearly, a more reasonable strategy is to maximize the AEP while constraining the mass using a surrogate of some kind. Unfortunately, choosing an appropriate surrogate constraint is not straightforward. One possibility is to constrain the root bending moment to be the same as the reference design. However, this is often not a helpful constraint. For example, if we define the problem as

$$
\begin{array}{ll}
\text { maximize } & A E P(x) \\
\text { with respect to } & x=\{\{c\},\{\theta\}, \lambda\} \\
\text { subject to } & V_{t i p}<V_{t i p_{\max }} \\
& M_{b \text { root }}<M_{\text {broot } 0}
\end{array}
$$

then the solution is exactly the same as if the root bending moment constraint was not included. This is because maximum AEP designs tend to decrease in root bending moment even without the constraint. Every point in Figure 10 actually has a lower root bending moment than the reference design. For example, Figure 12 compares the flapwise loads on the baseline design and design M1. The optimized design has larger loading inboard but decreased loading outboard; the net effect is that the root bending moment decreases slightly. Thus, including a root bending moment constraint would have no effect on the optimal solution.

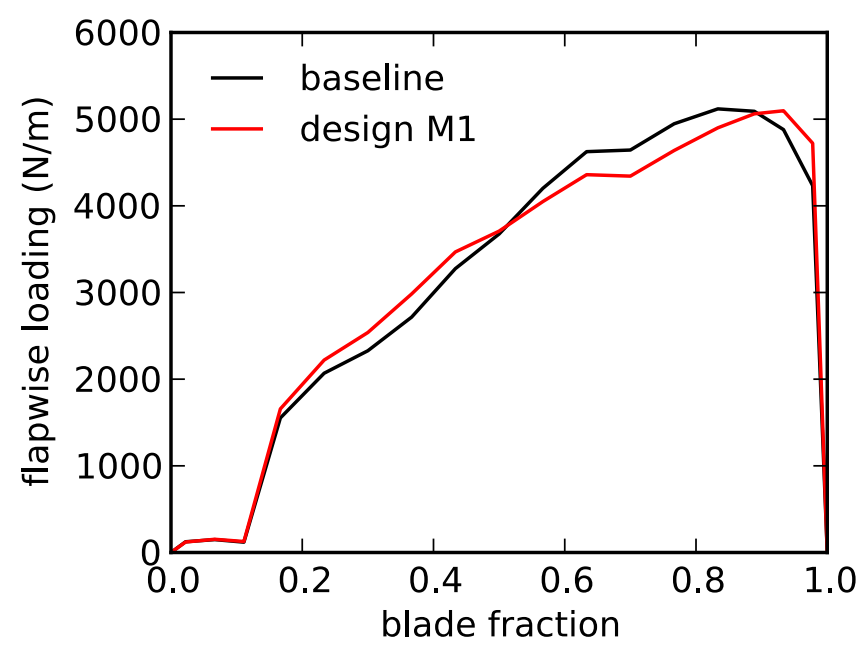

Figure 12. Flapwise loading for the baseline design and the maximum AEP solution with fixed mass (design M1). The root bending moment for the optimized design decreased, even without a root bending moment constraint.

A number of other potential surrogate constraints were evaluated. One of the more useful ones is summarized below. In conceptual aircraft design, it is common to consider wing weight as consisting of a portion that scales with the planform area and a portion that scales with the required loading. For the loading portion, we can approximate the bending moment at a section as a function of the local stress as

$$
M_{b}=\frac{\sigma A t}{2}
$$

where $A$ is the cross-sectional area of the structure required to resist the loads, and $t$ is the airfoil thickness. The mass of the material required to resist the bending loads is

$$
m=\int \rho A d r=2 \int \frac{\rho M_{b}}{\sigma t} d r
$$

If we make the approximation that density is uniform throughout the blade and the design is fully stressed, then the mass is proportional to the index

$$
m \propto i_{\text {aero }} \equiv \int \frac{M_{b}}{t} d r
$$


Although this approximation works well in aircraft design problems, ${ }^{41}$ the assumptions are more of a stretch for wind turbine blades. Blades typically use a composite structure that can vary significantly in effective density. Also, because of other constraints, the blade structure is generally not fully stressed. Even with these limitations, this metric is still likely to be more useful than root bending moment, as it captures the penalty of a shrinking structural box. Note that, while a uniform $t / c$ is often assumed for aircraft design, an estimate for the airfoil $t / c$ distribution is needed to evaluate this metric for wind turbines, as the variation is typically very significant.

Constraining the planform area $S_{\text {plan }}$ and the mass index $i_{\text {aero }}$ should help constrain the mass of the optimized design. If manufacturing or transportation limitations are known, a constraint on the maximum chord may be used instead of, or in addition to, a constraint on the area. However, with the root fatigue constraint in mind, these constraints alone often produce poor solutions. Typically, the optimal solution is to decrease the root chord in exchange for a larger chord at the point of maximum chord. This is slightly better aerodynamically, but is a considerable penalty structurally. To prevent this penalty, we added a constraint on the stress at the root of the blade. For a thin shell circular section at the blade root, the stress is equal to

$$
\sigma_{\text {root }}=\frac{M_{b} r}{\pi r^{3} t}
$$

where $r$ is the radius of the circular section and $t$ is the shell thickness. In this optimization problem, the internal structure was fixed so

$$
\sigma_{\text {root }} \propto \frac{M_{b}}{r^{2}}
$$

The "AEP 1st" optimization problem was done sequentially as follows

$$
\begin{array}{ll}
\text { maximize } & A E P(x) \\
\text { with respect to } & x=\{\{c\},\{\theta\}, \lambda\} \\
\text { subject to } & V_{\text {tip }}<V_{\text {tip }} \text { max } \\
& i_{\text {aero }}<i_{\text {aero } 0} \\
& S_{\text {plan }}<S_{\text {plan }_{0}} \\
& \left(M_{b} / r^{2}\right)_{\text {root }}<\left(M_{b} / r^{2}\right)_{\text {root }_{0}} \\
& m(x) \\
\text { minimize } & x=\{t\} \\
\text { with respect to } & c_{\text {set }}(x)<0 \\
\text { subject to } &
\end{array}
$$

Although this design achieved a relatively high AEP, it was not quite as high as the maximum $(0.39 \%$ increase compared to 0.56\%); however, it achieved this AEP with a slight decrease in blade mass (0.5\%). The net result was a $0.3 \%$ reduction in the cost of energy. Even though this is a much better solution than simply maximizing AEP, it is still far from a minimum cost of energy design. One of the main reasons for this is that, rather than simply avoiding a mass increase, the minimum cost of energy solution achieves a significant mass decrease. Because the blade shape is already dictated by the aerodynamic optimization, there is only so much that can be done to minimize mass by changing the internal structure. So when the aerodynamic and structural disciplines are coupled in shaping the blade, much greater net benefits are possible.

If the disciplines must be decoupled, an alternative approach is to allow the structural analysis to dictate the blade shape and the aerodynamic analysis to dictate the airfoil shape (only the twist distribution for this problem). Then the structural analysis must be repeated one final time to ensure that the constraints 
have been satisfied. This approach was used in the "mass 1st" problem, which was defined as

$$
\begin{aligned}
& \text { minimize } \quad m(x) \\
& \text { with respect to } x=\{\{c\},\{t\}\} \\
& \text { subject to } \quad c_{\text {set }}(x)<0 \\
& \text { maximize } \quad A E P(x) \\
& \text { with respect to } x=\{\{\theta\}, \lambda\} \\
& \text { subject to } \quad V_{t i p}<V_{t i p_{\max }} \\
& \text { minimize } \quad m(x) \\
& \text { with respect to } x=\{\{c\},\{t\}\} \\
& \text { subject to } \quad c_{\text {set }}(x)<0
\end{aligned}
$$

Minimizing mass, even when subject to the constraints, is not a particularly well-defined problem. The solution depends on the choice of variable bounds. In the "mass 1st" problem, the tip chord shrunk to the lower bound $(0.5 \mathrm{~m})$ and the position of the maximum chord $\left(r_{2}\right.$ in Figure $\left.4 \mathrm{a}\right)$ moved to its right bound ( $40 \%$ blade fraction). Moving to the extreme bounds allowed the optimizer to minimize mass near the root where the structure was heavy, while still keeping a large enough chord at the root to satisfy the fatigue constraint. For the "mass 1st" problem, the mass of the blades decreased significantly (6.6\%), but the AEP also decreased (0.9\%). There was a net decrease of $0.35 \%$ in cost of energy, but this is not a particularly useful design technique because the results are sensitive to the choice of bounds on the chord distribution.

We compared these designs to the real objective - the minimum cost of energy ("min COE") design

$$
\begin{array}{ll}
\text { minimize } & C O E(x) \\
\text { with respect to } & x=\{\{c\},\{\theta\},\{t\}, \lambda\} \\
\text { subject to } & c_{\text {set }}(x)<0
\end{array}
$$

The AEP, turbine mass, and cost of energy of the three optimized designs discussed in this section are compared in Figure 13. The "AEP 1st" design maximizes AEP first and then minimizes mass (Eq. (42)). The AEP optimization has constraints on the planform area and bending moment distribution. The "mass 1st" design minimizes mass first and then maximizes AEP (Eq. (43)). Changes in the chord distribution are dictated by the structural optimization. The real objective is to minimize the cost of energy and is shown in the final design "min COE" (Eq. (44)). Although only the rotor mass is optimized, the percent change in total turbine mass is shown in this figure (assuming constant mass values for the rest of the turbine). The mass of the rest of the turbine is added because the rotor mass changes by a relatively large percentage and would overwhelm the scale of the figure. The masses for the rest of the turbine come from those defined for the NREL 5-MW reference model ${ }^{32}\left(m_{\text {hub }}=56,780 \mathrm{~kg}, m_{\text {nacelle }}=240,000 \mathrm{~kg}\right.$ and $\left.m_{\text {tower }}=347,460 \mathrm{~kg}\right)$. Finally, the chord and twist distributions of the optimized designs are compared in Figure 14.

With the inclusion of reasonable constraints that help limit mass change, maximizing annual energy production by shaping the blade and subsequently minimizing mass by changing the internal structure can lead to designs that decrease the cost of energy. However, the impact is much smaller than it could be, because optimal designs tend to decrease mass until structural constraints become active. This is very difficult to approximate without including a structural model and corresponding integrated metric into the optimization.

On the other hand, with reasonable bounds on the design variables, minimizing the mass by changing the blade's external and internal structure and subsequently maximizing aerodynamic performance by changing the airfoils and tip-speed ratio can also lead to designs that decrease the cost of energy. However, aerodynamic performance suffers and the result is significantly suboptimal than designs that integrate the aerodynamic and structural analyses and use a relevant combined metric. 


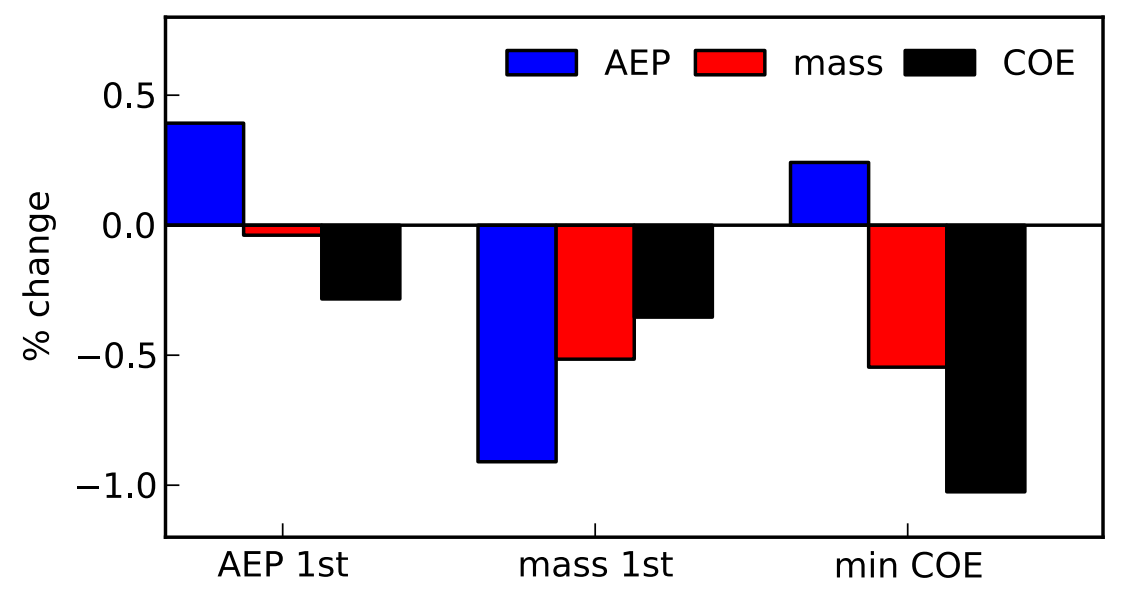

Figure 13. Comparison between sequential aerodynamic and structural optimizations and an integrated aerodynamic and structural optimization. The percent change in mass is relative to the total turbine mass.

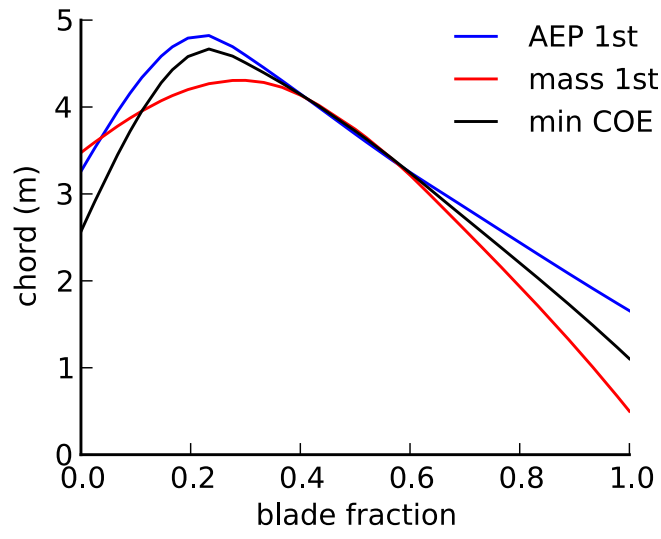

(a) Chord distribution along blade.

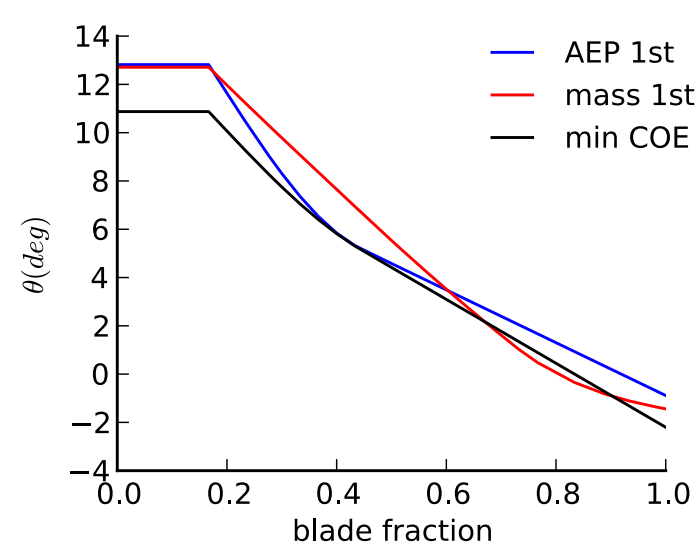

(b) Twist distribution along blade.

Figure 14. Chord and twist distribution for the three designs that were examined. 


\section{III.B. Minimum Turbine Mass/AEP}

Clearly, it is beneficial to directly integrate the aerodynamic and structural analyses in the optimization problem. Although maximizing AEP and minimizing mass sequentially is not particularly effective, an appropriate combined aero/structural metric can be useful. For designs with fixed materials, a reasonable choice is to minimize the ratio of the turbine mass to the annual energy production $m_{\text {turbine }} / A E P$. This objective can be motivated by the cost of energy equation, which includes capital costs and operating expenses in the numerator and AEP in the denominator

$$
\mathrm{COE}=\frac{\mathrm{FCR} \cdot(\mathrm{TCC}+\mathrm{BOS})+\mathrm{O} \& \mathrm{M}}{\mathrm{AEP}}
$$

In the absence of a detailed cost model, the assumption can be made that the capital costs and operating expenses will scale proportionally with the overall turbine mass.

For the remainder of this section, the mass of the turbine is simply denoted as $m$, rather than $m_{\text {turbine }}$. To compare the effectiveness of minimizing $m / A E P$ with minimizing COE, a number of studies were examined. The first study compared optimal turbines using both objectives at a fixed rotor diameter and a fixed machine rating $(5-\mathrm{MW})$. The rotor diameter was varied at several different values to observe the trends in performance. The optimization problem was given by

$$
\begin{array}{ll}
\text { minimize } & C O E(x ; D) \text { or } m(x ; D) / A E P(x ; D) \\
\text { with respect to } & x=\{\{c\},\{\theta\},\{t\}, \lambda\} \\
\text { subject to } & c_{\text {set }}(x)<0
\end{array}
$$

Although two separate objectives were used, for comparison purposes, both final designs were evaluated using the cost of energy metric. The difference in cost of energy between the two objectives as a function of rotor diameter is shown in Figure 15a, and the variation in $m / A E P$ is shown in Figure 15b.

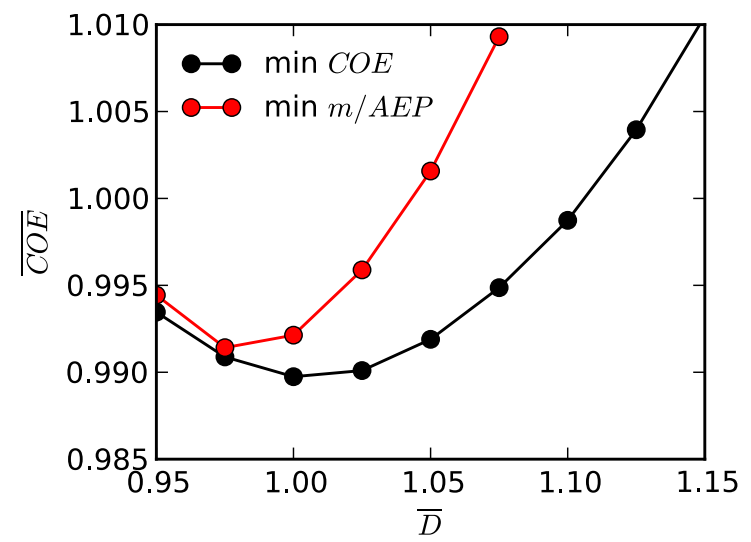

(a) Solutions are compared to minimum cost of energy solutions (both evaluated using $\mathrm{COE}$ ).

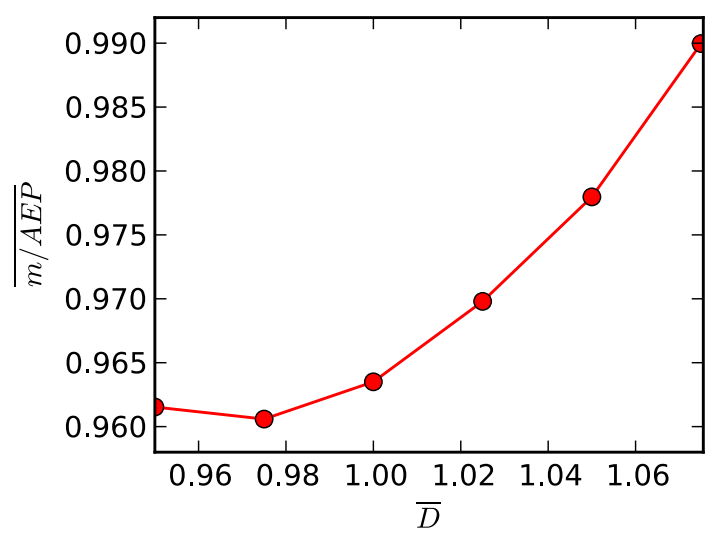

(b) Variation in $m / A E P$ approximately predicts the correct optimal diameter.

Figure 15. Designs with minimum $m / A E P$ as a function of rotor diameter.

The metric $m / A E P$ resulted in suboptimal designs in terms of minimum COE, though they were the optimal solution for minimum $m / A E P$. Both metrics found very similar designs for diameters smaller than the reference design, but for larger diameter rotors, the minimum $m / A E P$ designs had about a $1 \%$ higher cost of energy. The reason for this discrepancy is that the metric $m / A E P$ overemphasizes the role of the tower mass. Figure 16a shows the relative contribution to total mass from the different components of the turbine and Figure 16b shows the relative contribution to total cost for the baseline design. The tower dominates the mass of the turbine at about $53 \%$. However, it contributes a much smaller fraction to the total costs (about $9 \%$ ). These relative contributions are typical of land-based turbines. ${ }^{42}$ Thus, when the objective is to minimize $m / A E P$, the contribution of the tower plays a disproportionate role. At least, a fairly accurate optimal rotor diameter is predicted using this metric (Figure 15b), which is not always the case with $m / A E P$ metrics. 
The difference between the minimum $\mathrm{COE}$ and minimum $m / A E P$ designs can be seen more clearly by examining the chord and twist distributions as the rotor diameter increases. Figure 17 compares the optimal chord and twist distribution for the two metrics at $D / D_{0}=1.05$. The minimum $m / A E P$ design attempts to sacrifice rotor performance to reduce thrust and decrease tower mass. We observe that the minimum $m / A E P$ design uses a thinner structure outboard to reduce thrust (and power) and unloads the tip significantly to further reduce thrust. To compensate for the reduced structure, the design has a much higher spar cap thickness outboard. Compared to the minimum COE design, the minimum $m / A E P$ design produces $7.7 \%$ less thrust at rated speed, which allows for a $6.7 \%$ lighter tower. At the same time, the annual energy production decreases by $1.9 \%$. Because the contribution of the tower mass is so large, the net effect is still a reduction in $m / A E P$. In this case, minimizing $m / A E P$ is not a good surrogate for minimizing the cost of energy (though it is better than just maximizing AEP).

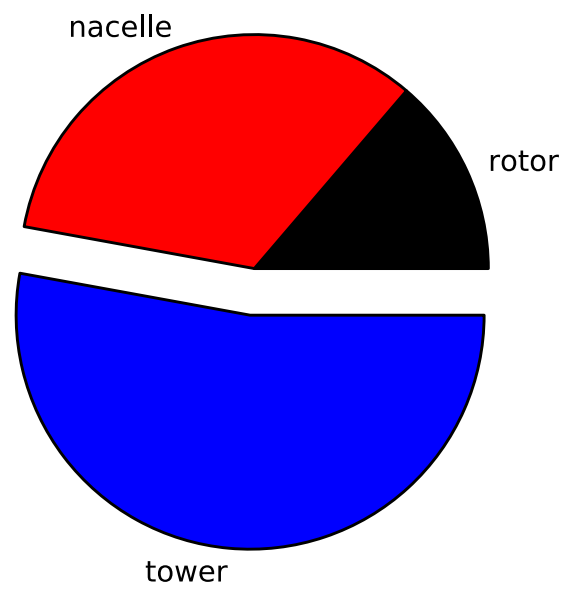

(a) Relative contribution to total mass.

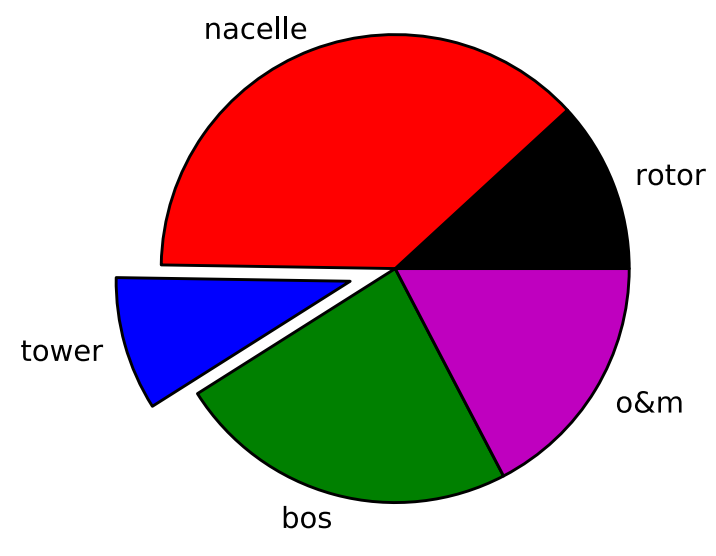

(b) Relative contribution to total cost.

Figure 16. Relative contributions to total mass and total cost of the baseline design. Cost contributions already include the fixed charge rate and tax rate. The tower contribution is of particular note.

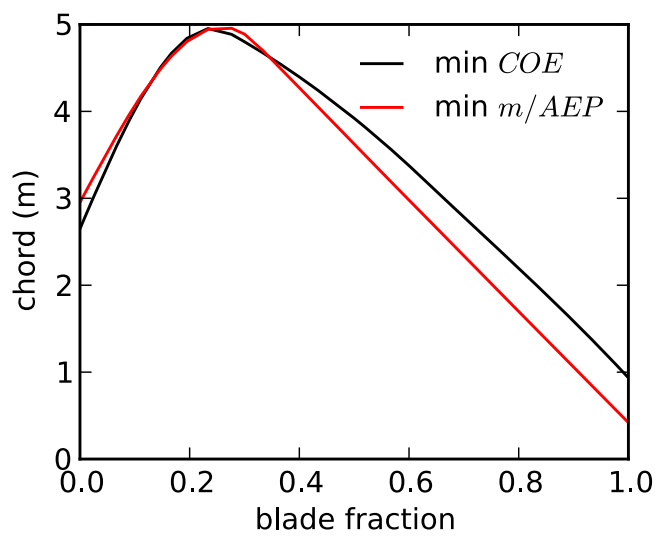

(a) Comparison between chord distributions.

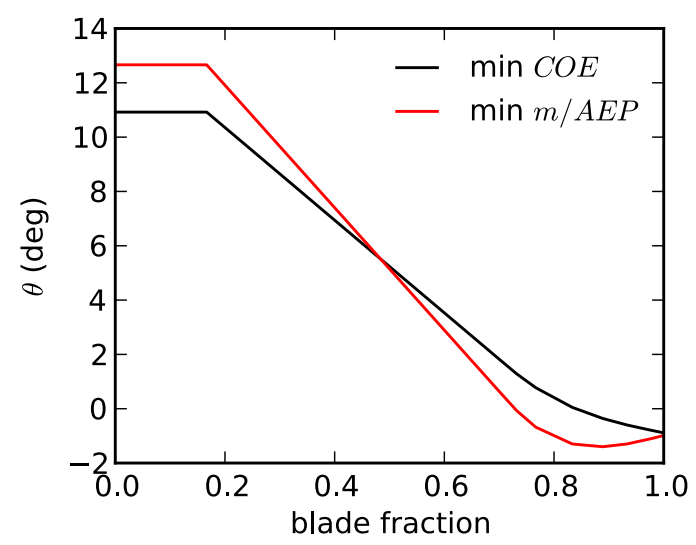

(b) Comparison between twist distributions.

Figure 17. Comparison of chord and twist distributions for minimum COE and minimum $m / A E P$ design at $D / D_{0}=1.05$.

To improve the predictive ability of $m / A E P$, other alternatives may be considered. For example, if tower mass is disproportionately emphasized in minimizing $m / A E P$, perhaps the tower mass could be fixed. 
However, ignoring the tower completely and minimizing $m_{\text {rotor }} / A E P$ is not a useful option. Similar to the previous example, this metric overemphasizes the effect of the rotor mass and thus leads to optimal solutions that reduce rotor mass at great expense to aerodynamic performance. Instead, including a fixed tower and nacelle mass is a much more reasonable approach. We defined

$$
m_{\text {fixed }}=m_{\text {blades }}+m_{\text {other }}
$$

where the mass of the blades was estimated as before, but the contribution of the other components were fixed at the values reported for the NREL 5-MW reference model ${ }^{32}\left(m_{\text {hub }}=56,780 \mathrm{~kg}, m_{\text {nacelle }}=240,000\right.$ $\mathrm{kg}$, and $\left.m_{\text {tower }}=347,460 \mathrm{~kg}\right)$. The optimization problem was then

$$
\begin{array}{ll}
\text { minimize } & m_{\text {fixed }}(x ; D) / A E P(x ; D) \\
\text { with respect to } & x=\{\{c\},\{\theta\},\{t\}, \lambda\} \\
\text { subject to } & c_{\text {set }}(x)<0
\end{array}
$$

The minimum $m_{\text {fixed }} / A E P$ designs are compared to the minimum $\mathrm{COE}$ designs as a function of rotor diameter in Figure 18a. Although the first design was not optimized for minimum COE, they were both evaluated for cost of energy to facilitate a comparison. We note that this objective produced essentially the same designs as the minimum COE designs, which is somewhat fortuitous. The constant nacelle and tower masses played a fractional role that was similar to the role of the balance-of-station (BOS) and operations and maintenance costs in estimating cost of energy (though they were not precisely constant). Figure 16 shows that the rotor mass and rotor costs consume similar fractions of total mass and total cost, respectively.

However, there is still a problem with the metric $m_{\text {fixed }} / A E P$. The use of this metric implies that a cost of energy estimate is absent. Figure $18 \mathrm{~b}$ shows the variation in $m_{\text {fixed }} / A E P$ as a function of rotor diameter. We see that the trend is completely opposite to that of the cost of energy. Although the trend may not always reverse, the relative change between designs will certainly differ between the two metrics. As the rotor diameter increases, the annual energy production decreases faster than $m_{\text {fixed }}$ increases. The difference is because only the rotor mass is changing in $m_{\text {fixed }}$, and it is a relatively small component of the total mass. On the other hand, as the rotor diameter increases, turbine capital costs generally increase at a much faster rate than $m_{\text {fixed }}$ (BOS and operations and maintenance costs also increase somewhat). These results suggest that $m_{\text {fixed }} / A E P$ may work well for fixed diameter optimizations, but for variable diameter studies, this metric will not predict the correct rotor diameter, and may, in fact, suggest very different optimal diameters.

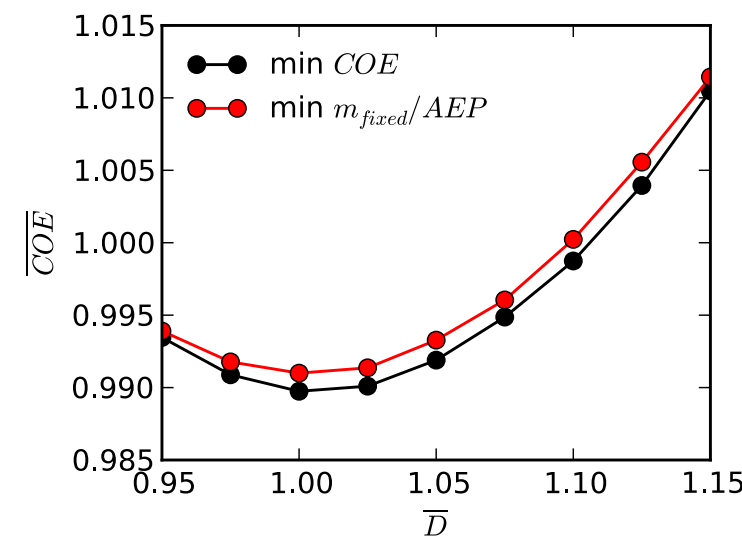

(a) Solutions are compared to minimum cost of energy solutions (both evaluated using COE).

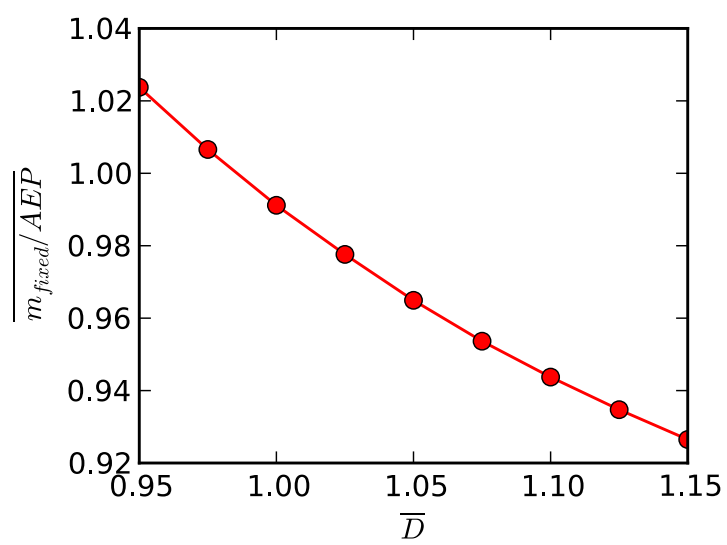

(b) Variation in $m_{\text {fixed }} / A E P$ predicts wrong trend.

Figure 18. Designs with minimum $m_{\text {fixed }} / A E P$ as a function of rotor diameter.

One final modification to the $m / A E P$ metric was made. It was clear that, in order to capture the correct variation in performance as rotor diameter changed, the change in the nacelle and tower mass must be estimated. However, this must be done in such a way that the optimizer will not have an overly large incentive to reduce tower mass at the expense of aerodynamic performance. As discussed in Section II.C, 
the tower sizing scaled primarily with rotor thrust. In Eq. (25), the thrust contribution to the tower's axial stress is generally much larger than from the weight of the top mass. We assumed that tower mass scaled directly with the tower thrust. This is the type of trend seen in WindPACT studies and in the NREL cost and scaling model. ${ }^{10}$ Therefore, we can estimate the mass about a reference design as

$$
\frac{m}{m_{0}}=\frac{C_{T} V_{\text {rated }}^{2} D^{2}}{C_{T_{0}} V_{\text {rated }}^{2} D_{0}^{2}}
$$

This equation allowed us to estimate tower mass as rotor diameter changed (conservatively) but, as discussed previously for the objective minimum $m / A E P$, the optimal solution significantly decreased rotor thrust (and thus power) to reduce tower mass. This same incentive exists here by decreasing the thrust coefficient of optimized designs. To avoid this incentive, we removed thrust coefficient from the equation, with the assumption that optimal solutions should have similar maximum thrust coefficients. Then the mass can be estimated as

$$
\frac{m}{m_{0}}=\frac{\left(V_{\text {rated }} D\right)^{2}}{\left(V_{\text {rated }} D\right)_{0}^{2}}
$$

For a given rotor diameter, the only way for the optimization to decrease the tower mass is to decrease rated speed. Because the optimization was done at a fixed machine rating, it was synonymous with increasing aerodynamic performance. Thus, with this "trick" we aligned the objectives and forced the optimizer to find reasonable designs. We will denote this mass calculation as $m_{\text {modified }}$, where all mass components are estimated in the same way, except for tower mass, which was estimated using

$$
\frac{m_{\text {tower-modified }}}{m_{\text {tower-modified } 0}}=\max \left(\sqrt{0.9}, \frac{\left(V_{\text {rated }} D\right)^{2}}{\left(V_{\text {rated }} D\right)_{0}^{2}}\right)
$$

where the first portion came from Section II.C to account for a constraint on the moment of inertia, and a cubic spline was added to provide a smooth transition in the tower mass.

Figure 19a shows a comparison in cost of energy between designs optimized with the modified mass metric and minimum cost of energy designs. The designs differed only slightly, with the minimum $m_{\text {modified }} / A E P$ designs differ only by about $0.3 \%$ from the optimum cost of energy. However, like the previous case, this metric does not predict the correct optimal rotor diameter (Figure 19b).

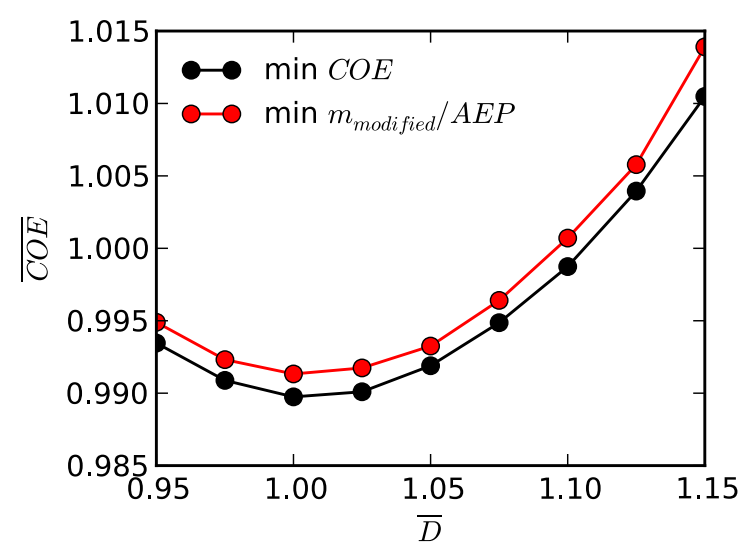

(a) Solutions are compared to minimum cost of energy solutions (both evaluated using COE).

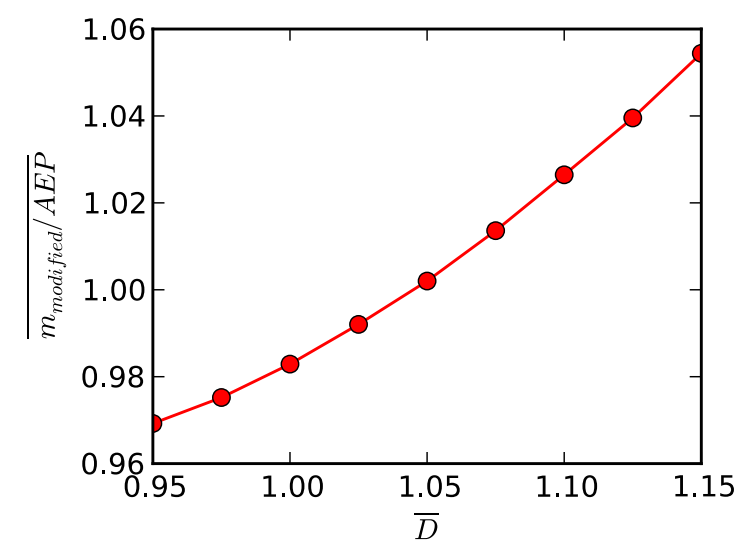

(b) Variation in $m_{\text {modified }} / A E P$ does not predict the correct optimal rotor diameter.

Figure 19. Designs with minimum $m_{\text {modified }} / A E P$ as a function of rotor diameter.

We have seen that $m / A E P$ can be a useful metric for designs with a fixed rotor diameter and power rating, but the optimization must be set up carefully. Because the mass of the tower is such a large portion of turbine mass, and tower cost is not nearly as large of a fraction of total cost, the problem must be carefully designed to prevent the optimizer from taking advantage of this difference. One approach is to fix the nacelle and tower mass at constant values. This does lead to good designs at a fixed rotor diameter, but is a poor metric for variable-diameter problems, as the turbine mass changes much more slowly than it should as the 
rotor diameter changes. At the same time, including an estimate of tower mass will often incentivize the optimizer to sacrifice aerodynamic performance to reduce tower mass. If the optimization problem is set up in such a way that this incentive is prevented, then reasonably good designs (close to minimum COE designs) can be found for a given diameter. However, predicting the optimal diameter may still lead to incorrect conclusions.

\section{III.C. Minimum Cost of Energy}

Minimizing $m / A E P$ is certainly not a useful metric if the machine rating is allowed to vary. If a tower model is included, then there is a large incentive to reduce the rating to minimize the tower mass. If the tower mass is fixed, then there is little to no penalty for increasing the rating all the way up to its upper bound. A cost model is necessary to capture costs associated with the machine rating, and to appropriately scale the impact of the tower costs with the rest of the costs.

However, the details of the cost model cannot be an afterthought. Care must be taken to capture the important effects. Often, optimization studies put considerable effort into improving the fidelity of the physics modeling, but comparatively little effort into improving the fidelity of the cost model. As an example, a similar study to the previous section was undertaken, but with variable machine rating

$$
\begin{array}{ll}
\text { minimize } & C O E(x ; \text { rating }) \\
\text { with respect to } & x=\{\{c\},\{\theta\},\{t\}, \lambda, D\} \\
\text { subject to } & c_{\text {set }}(x)<0
\end{array}
$$

The optimization was done at a fixed power output of the plant, with a baseline of fifty 5-MW turbines. Only the new BOS model is sensitive to changes in the number of turbines, the other components of the cost equation are currently invariant to this change. Figure 20 compares the minimum cost of energy design as a function of rating using the newly developed BOS model, and the original BOS model from the cost and scaling model. Clearly, there is a substantial difference in the optimal machine rating (about 30\%), and a significant difference in optimal designs at a fixed rating. The only difference in these curves is the BOS model. The previous BOS model uses simple scaling relationships (that do not scale well to large machine ratings), while the new one uses a bottom-up approach in estimating component sizes and quantity. Thus, even though improved fidelity exists in the physics, it is also needed in the cost modeling to obtain quality solutions.

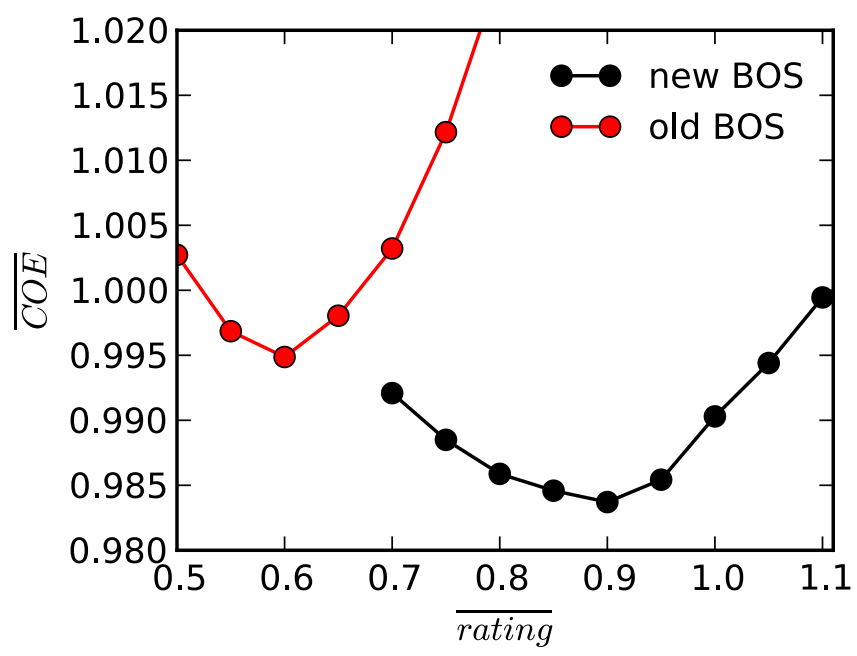

Figure 20. Comparison of minimum cost of energy designs as a function of machine rating using both the old and new BOS model.

Though cost of energy is clearly a superior metric, a deterministic model may still be insufficient to produce robust designs. One simple example is given below. Consider that all designs have been optimized for a fixed wind speed distribution (Rayleigh distribution with a mean wind speed of $10 \mathrm{~m} / \mathrm{s}$ ). However, a 
manufacturer may design a turbine to be used at many sites. We considered a problem where the turbine was intended for use in any wind power class from 3 to 7 , which is common, and where the mean wind speed at $50 \mathrm{~m}$ above the ground varies from $6.4-11.9 \mathrm{~m} / \mathrm{s}$. The turbine was optimized to minimize the expected value of the cost of energy across those wind speeds, and the 50-m wind speed was used as a proxy for the hub height wind speed. We gave each wind speed an equal weight (uniform distribution), though any distribution could be used. The optimization problem was defined as

$$
\begin{array}{ll}
\operatorname{minimize} & <\operatorname{COE}\left(x ; \bar{V}_{h u b}\right)> \\
\text { where } & \bar{V}_{h u b} \sim \mathcal{U}(6.4,11.9) \\
\text { with respect to } & x=\{\{c\},\{\theta\},\{t\}, \lambda, \text { D, rating }\} \\
\text { subject to } & c_{\text {set }}(x)<0
\end{array}
$$

The cost of energy as a function of wind speed is shown for the optimal solution as compared to the point design, which minimizes COE at $\bar{V}_{h u b}=10 \mathrm{~m} / \mathrm{s}$ in Figure 21. While the point design has a slightly lower cost of energy at the design point of $V_{h u b}=10 \mathrm{~m} / \mathrm{s}$, the robust design has a much lower cost of energy at the lower wind speeds. The optimal blade shapes are similar; the main difference is that the robust design has a $16 \%$ lower machine rating to achieve a higher capacity factor at the lower wind speeds. This also allows the robust design to use a $3.4 \%$ larger radius and still satisfy the structural constraints. The net result is that the robust design achieves a $1.2 \%$ lower average cost of energy than the point design. Although there are clear benefits to site-specific design, even within a single site the variation in wind conditions may have a considerable impact.

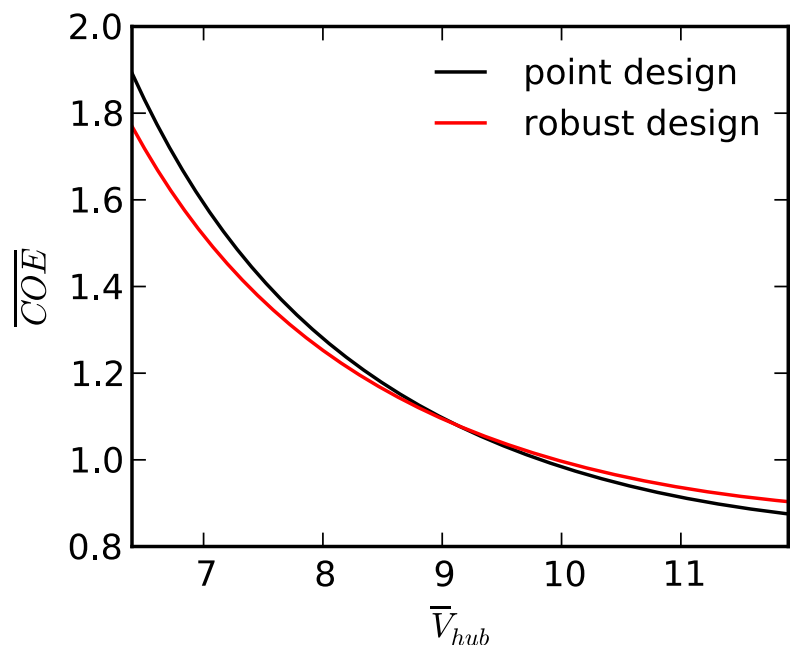

Figure 21. Cost of energy as a function of hub wind speed for a point design that was optimized at $10 \mathrm{~m} / \mathrm{s}$, as compared to a robust design that was optimized to minimize the expected value of the cost of energy across the wind speeds.

This example may be simplistic, but it demonstrates the importance of considering multiple design points in an optimization for improved robustness. Structural sizing constraints are affected by uncertainty associated with the loading conditions, safety factors, etc., while performance metrics are affected by variability in the wind speed distributions, availability, etc. Characterizing the uncertainty of the model inputs and performing optimizing under uncertainty is important to achieving robust designs.

\section{Conclusion}

In this paper, we discussed the limitations of various objectives that are commonly used in wind turbine optimization problems. The specific optimized designs produced in this study are not of primary interest, as they will vary depending on the assumptions and fidelity of the model. However, the relative differences in performance between different objectives highlight the following fundamental conclusions. 
First, maximizing annual energy production typically leads to significantly suboptimal designs (in terms of cost of energy), even when the internal structure is subsequently optimized. Part of the difficulty is that similar aerodynamic performance can be achieved with designs that have very different masses. Appropriate aerodynamic surrogates for mass and structural limitations are helpful, but are still considerably inferior to a true structural model. Shaping the blade to minimize mass and subsequently optimizing airfoil sections for maximum aerodynamic performance is not much better. Although both approaches lead to decreases in cost of energy, they are inferior to metrics that combine the aerodynamic and structural performance.

Second, minimizing the ratio of turbine mass to annual energy production can be a useful metric, but only for certain design problems and only if used with care. Even if a nacelle and tower model are not used, a constant estimate of their mass must be included, otherwise potential decreases in rotor mass are overemphasized, which can lead to extremely suboptimal aerodynamic performance. As the rotor diameter (and thrust) change, exactly how the tower mass is estimated can substantially affect the optimal result. If a fixed tower mass is used, then the optimization works well at a fixed rotor diameter, but for a variablediameter design, it predicts a very inaccurate optimal diameter. This error arises because, without changing the size of the nacelle or drivetrain, the ratio of turbine mass to annual energy production decreases much faster than it otherwise should. On the other hand, when the tower is allowed to resize, the problem must be constructed very carefully. The difficulty is that tower mass consists of a large portion of total mass, but tower cost is a rather small contribution to total cost. Thus, using $m / A E P$ as the objective significantly overemphasizes the role of the tower. Without careful construction of the problem, the objective $m / A E P$ overincentivizes the optimizer to decrease tower mass at the expense of aerodynamic performance. With carefully constrained designs, this metric can work quite well for a fixed rotor diameter, but it may still lead to incorrect optimal diameters for variable-diameter designs. For variable machine rating problems (or designs where the material is varied), this metric is not helpful at all.

Finally, minimum cost of energy is the appropriate metric to balance aerodynamic and structural performance with plant-level and operational costs. However, the fidelity of the cost model can dramatically affect the results. Along with increased fidelity in the physics, increased fidelity in cost modeling is needed. Furthermore, simply minimizing cost of energy may not be the most appropriate metric, as turbines need to be designed to perform well in a variety of conditions. Even within a single site, environmental conditions may vary significantly. Many of these inputs are inherently stochastic, and uncertainty exists in operational and model parameters. These considerations suggest that simply minimizing cost of energy will lead to inferior designs, and that optimization under uncertainty is particularly important given the stochastic nature of the wind.

This paper has highlighted many of the important design considerations in choosing appropriate objectives for wind turbine optimization problems. However, many opportunities exist to improve upon the insights discussed here. Rather than using scaling relationships, full physics-based models for the drivetrain and tower can be used. This will lend additional degrees of freedom to the optimization problem, allow for more rigorous sizing constraints, and lead to a better understanding of the trade-offs in rotor aerodynamic performance and turbine weight. The capital cost models used in this study are very simplistic; more detailed cost models are needed to better capture the effect of materials and manufacturing costs that are the result of changes to the structural ply schedule and blade shape. More thorough studies combining optimization with uncertainty quantification are needed, along with a better understanding of the nature of the uncertainties associated with the environmental conditions, physical processes, and cost metrics. Some of these potential improvements are currently being investigated.

\section{Acknowledgments}

The authors gratefully acknowledge George Scott of NREL for providing a Python version of NREL's cost and scaling model. This work was supported by the U.S. Department of Energy under Contract No. DE-AC36-08GO28308 with the National Renewable Energy Laboratory.

\section{References}

\footnotetext{
${ }^{1}$ Fuglsang, P. and Madsen, H., "Optimization Method for Wind Turbine Rotors," Journal of Wind Engineering and Industrial Aerodynamics, Vol. 80, 1999, pp. 191-206.

${ }^{2}$ Diveux, T., Sebastian, P., Bernard, D., Puiggali, J., and Grandidier, J., "Horizontal Axis Wind Turbine Systems: Optimization Using Genetic Algorithms," Wind Energy, Vol. 4, 2001, pp. 151-171.
} 
${ }^{3}$ Fuglsang, P. and Thomsen, K., "Site-Specific Design Optimization of 1.5-2.0 MW Wind Turbines," Journal of Solar Energy Engineering, Vol. 123, No. 4, 2001, pp. 296.

${ }^{4}$ Fuglsang, P., Bak, C., Schepers, J., Bulder, B., Cockerill, T., Claiden, P., Olesen, A., and van Rossen, R., "Sitespecific Design Optimization of Wind Turbines," Wind Energy, Vol. 5, No. 4, 2002, pp. 261-279.

${ }^{5}$ Kenway, G. and Martins, J., "Aerostructural Shape Optimization of Wind Turbine Blades Considering Site-Specific Winds," Proc. of 12th AIAA/ISSMO Multidisciplinary Analysis and Optimization Conference, Victoria, British Columbia, Canada, September 2008.

${ }^{6}$ Petrone, G., de Nicola, C., Quagliarella, D., Witteveen, J., Axerio-Cilies, J., and Iaccarino, G., "Wind Turbine Optimization Under Uncertainty with High Performance Computing," AIAA Applied Aerodynamics Conference, AIAA 2011-3806, Honolulu, HI, June 2011.

${ }^{7}$ Maki, K., Sbragio, R., and Vlahopoulos, N., "System Design of a Wind Turbine Using a Multi-Level Optimization Approach," Renewable Energy, Vol. 43, No. 2012, 2011, pp. 101-110.

${ }^{8}$ Bottasso, C., Campagnolo, F., and Croce, A., "Computational Procedures for the Multi-Disciplinary Constrained Optimization of Wind Turbines," Scientific Report DIA-SR, January 2010, pp. 10-02.

${ }^{9}$ Dykes, K., Meadows, R., Felker, F., Graf, P., Hand, M., Lunacek, M., Michalakes, J., Moriarty, P., Musial, W., and Veers, P., "Applications of Systems Engineering to the Research, Design, and Development of Wind Energy Systems," Tech. Rep. NREL/TP-5000-52616, National Renewable Energy Laboratory, December 2011.

${ }^{10}$ Fingersh, L., Hand, M., and Laxson, A., "Wind Turbine Design Cost and Scaling Model," Tech. Rep. NREL/TP-50040566, National Renewable Energy Laboratory, Golden, CO, December 2006.

${ }^{11}$ McWilliam, M. and Crawford, C., "The Behavior of Fixed Point Iteration and Newton-Raphson Methods in Solving the Blade Element Momentum Equations," Wind Engineering, Vol. 35, No. 1, 2011, pp. 17-32.

${ }^{12}$ Masters, I., Chapman, J., Orme, J., and Willis, M., "A Robust Blade Element Momentum Theory Model for Tidal Stream Turbines Including Tip and Hub Loss Corrections," Proceedings of IMarEST-Part A-Journal of Marine Engineering and Technology, Vol. 10, No. 1, 2011, pp. 25-35.

${ }^{13}$ Ning, S. A., "A Simple Solution Method for the Blade Element Momentum Equations with Guaranteed Convergence," Wind Energy, 2013, to appear.

${ }^{14}$ Brent, R. P., "An Algorithm with Guaranteed Convergence for Finding a Zero of a Function," The Computer Journal, Vol. 14, No. 4, 1971, pp. 422-425.

${ }^{15}$ Glauert, H., Airplane Propellers, Vol. 4, Springer Verlag, 1935.

${ }^{16}$ Buhl Jr., M. L., "A New Empirical Relationship between Thrust Coefficient and Induction Factor for the Turbulent Windmill State," NREL/TP-500-36834, National Renewable Energy Laboratory, Golden, CO, August 2005.

${ }^{17} \mathrm{Du}, \mathrm{Z}$. and Selig, M., "A 3-D Stall-Delay Model for Horizontal Axis Wind Turbine Performance Prediction," 1998 ASME Wind Energy Symposium, No. AIAA-1998-21, Jan 1998.

${ }^{18}$ Eggers Jr, A. J., Chaney, K., and Digumarthi, R., "An Assessment of Approximate Modeling of Aerodynamic Loads on the UAE Rotor," Aerospace Sciences Meeting and Exhibit, No. AIAA-2003-0868, Jan 2003.

${ }^{19}$ Viterna, L. and Janetzke, D., "Theoretical and Experimental Power from Large Horizontal-Axis Wind Turbines," NASA TM-82944, National Aeronautics and Space Administration, Cleveland, OH. Lewis Research Center, September 1982.

${ }^{20}$ Maples, B., Hand, M., and Musial, W., "Comparative Assessment of Direct Drive High Temperature Superconducting Generators in Multi-Megawatt Class Wind Turbines," Tech. Rep. NREL/TP-5000-49086, National Renewable Energy Laboratory, October 2010.

${ }^{21}$ Bywaters, G., John, V., Lynch, J., Mattila, P., Norton, G., Stowell, J., Salata, M., Labath, O., Chertok, A., and Hablanian, D., "Northern Power Systems WindPACT Drive Train Alternative Design Study Report," Tech. Rep. NREL/SR500-35524, National Renewable Energy Laboratory, October 2004.

${ }^{22}$ International Electrotechnical Commission, "International Standard IEC 61400-1," Wind turbine generation systems, 2005.

${ }^{23}$ Yang, T. Y., Finite Element Structural Analysis, Prentice-Hall, 1986.

${ }^{24}$ Bir, G., "User's Guide to PreComp," Tech. rep., National Renewable Energy Laboratory, September 2005.

${ }^{25}$ Hansen, M. O. L., Aerodynamics of Wind Turbines, Earthscan, 2nd ed., 2008.

${ }^{26}$ Halpin, J. C., Primer on Composite Materials Analysis, Technomic, 2nd ed., 1992.

${ }^{27}$ Griffith, D. T. and Ashwill, T. D., "The Sandia 100-Meter All-Glass Baseline Wind Turbine Blade: SNL100-00," SAND2011-3779, Sandia National Laboratories, Albuquerque, June 2011.

${ }^{28}$ Bir, G. S., "Computerized Method for Preliminary Structural Design of Composite Wind Turbine Blades," Transactions of the ASME, Vol. 123, No. 4, 2001, pp. 372-381.

${ }^{29}$ Mandell, J. and Samborsky, D., "DOE/MSU Composite Material Fatigue Database: Test Methods, Materials, and Analysis," Tech. Rep. Contractor Report SAND97-3002, Sandia National Laboratories, Albuquerque, NM, 1997.

${ }^{30}$ Dykes, K., "Development of Wind Turbine Component Mass-based Cost Models," NREL Technical Report (not yet published).

${ }^{31}$ Maples, B., Hand, M., and Saur, G., "Land-Based Wind Plant Balance of Station Cost and Scaling Model," NREL Technical Report (not yet published).

32 Jonkman, J., Butterfield, S., Musial, W., and G., S., "Definition of a 5-MW Reference Wind Turbine for Offshore System Development," Tech. Rep. NREL/TP-500-38060, National Renewable Energy Laboratory, Golden, CO, Feb 2009.

${ }^{33}$ Lindenburg, C., "Aeroelastic Modeling of the LMH64-5 Blade," Tech. Rep. DOWEC-02-KL-083/0, DOWEC 10083_001, Energy Research Center of the Netherlands, December 2002.

${ }^{34}$ Kooijman, H., Lindenburg, C., Winkelaar, D., and van der Hooft, E., "DOWEC 6 MW Pre-Design: Aero-Elastic Modeling of the DOWEC 6 MW Pre-Design in Phatas," Tech. Rep. ECN-CX-01-135, DOWEC 10046_009, Energy Research Center of the Netherlands, September 2003. 
${ }^{35}$ Brian Resor, S. N. L., "Definition of a 61.5-Meter Wind Turbine Blade Reference Model," personal communication regarding a draft Sandia National Laboratories report, 2012.

${ }^{36}$ Berg, J. and Resor, B., "Numerical Manufacturing and Design Tool (NuMAD V2.0) for Wind Turbine Blades: User's Guide," Tech. Rep. SAND2012-728, Sandia National Laboratories, Albuquerque, NM, 2012.

${ }^{37}$ Akima, H., "A New Method of Interpolation and Smooth Curve Fitting Based on Local Procedures," Journal of the ACM, Vol. 17, No. 4, October 1970, pp. 589-602.

${ }^{38}$ Martins, J., Sturdza, P., and Alonso, J., "The Complex-Step Derivative Approximation," ACM Transactions on Mathematical Software (TOMS), Vol. 29, No. 3, 2003, pp. 245-262.

${ }^{39}$ Fike, J. and Alonso, J., "Automatic Differentiation through the Use of Hyper-Dual Numbers for Second Derivatives," Recent Advances in Algorithmic Differentiation, 2012, pp. 163-173.

${ }^{40}$ Burton, T., Jenkins, N., Sharpe, D., and Bossanyi, E., Wind Energy Handbook, Wiley, 2011.

${ }^{41}$ Ning, S. A. and Kroo, I., "Multidisciplinary Considerations in the Design of Wings and Wing Tip Devices," Journal of Aircraft, Vol. 47, No. 2, 2010, pp. 534.

${ }^{42}$ Tegen, S., Hand, M., Maples, B., Lantz, E., Schwabe, P., and Smith, A., "2010 Cost of Wind Energy Review," Tech. Rep. NREL/TP-5000-52920, National Renewable Energy Laboratory, Golden, CO, April 2012. 